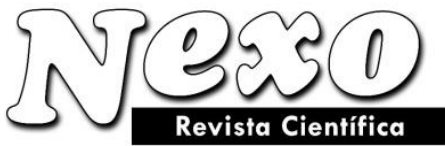

ISSN-E 1995-9516

Universidad Nacional de Ingeniería COPYRIGHT @ (UNI). TODOS LOS DERECHOS RESERVADOS http://revistas.uni.edu.ni/index.php/Nexo https://doi.org/10.5377/nexo.v33i02.10792

\title{
Mineral chemistry and thermo-barometry of intrusives from northern side of Shahroud river around Zardkuh in Guilan province (northern Iran)
}

\section{Infiltraciones químicas y de temperatura y presión barométrica en el lado norte del río Shahroud cerca de Zardkooh en la provincia de Gilan (norte de Irán)}

\author{
Mojtaba Bahajrouy, Saeed Taki*, Alireza Ganji \\ Department of Geology, Faculty of Basic Sciences, Lahijan Branch, Islamic Azad University, \\ Lahijan, Iran. \\ *Corresponding author email: taki_saeed2002@yahoo.com
}

(recibido/received: 01-July-2020; aceptado/accepted: 15-September-2020)

\begin{abstract}
The study area is located in northern Iran and is part of the Alborz mountain range. The exposed rock units in the study area are mostly Eocene volcanic rocks with some Oligomiocene intrusive masses included and locally Paleocene, Jurassic, Permian and Carboniferous sedimentary outcrops. The intrusive rocks of the study area are mostly gabbro and olivine gabbro in terms of mineralogical composition, but intermediate types such as porphyry monzonite are also rarely seen. Based on the results of microprobe analysis, pyroxenes have augite and diopside compositions, orthopyroxenes are hypersthenes, biotites have magnesium biotite and phlogopite compositions, olivines have hyalosiderite compositions and amphiboles are pargasite and ferropargasite compositionally. The results of temperature and pressure evaluation indicate a temperature range of 650 to $1200^{\circ} \mathrm{C}$ and pressure range $>2-5 \mathrm{~KB}$. Based on the chemistry of clinopyroxene mineral of the mentioned intrusions, magma that made these intrusions is of the sub-alkaline type and is formed in a volcanic arc tectonic setting.
\end{abstract}

Keywords: Mineral Chemistry, Thermometry and Barometry, Intrusive Mass, Volcanic Arc, Shahroud River, Alborz

\section{RESUMEN}

El área de estudio se encuentra en el norte de Irán y es parte de la cordillera de Alborz. Las unidades de roca expuestas en el área de estudio son en su mayoría rocas volcánicas del Eoceno con algunas masas intrusivas del Oligomioceno incluidas y afloramientos sedimentarios localmente del Paleoceno, Jurásico, Pérmico y Carbonífero. Las rocas intrusivas del área de 
estudio son en su mayoría gabro y gabro olivino en términos de composición mineralógica, pero los tipos intermedios como pórfido monzonita también se ven raramente. Según los resultados del análisis de microsonda, los piroxenos tienen composiciones de augita y diópsido, los ortopiroxenos son hiperstenos, las biotitas tienen composiciones de biotita y flogopita de magnesio, las olivinas tienen composiciones de hialosiderita y los anfíboles son pargasita y ferropargasita en su composición. Los resultados de la evaluación de temperatura y presión indican un rango de temperatura de 650 a $1200^{\circ} \mathrm{C}$ y un rango de presión> 2-5 KB. Con base en la química del mineral clinopiroxeno de las intrusiones mencionadas, el magma que hizo estas intrusiones es de tipo subalcalino y se forma en un entorno tectónico de arco volcánico.

Palabras clave: Química Mineral, Temperatura y Barómetro, Masa de infiltración, Arco volcánico, Río Shahroud, Alborz

\section{INTRODUCTION}

The nature of magma has a direct effect on the chemical composition of minerals, so minerals are able to retain and provide valuable informations about magmatic processes (Hou et al., 2015; Liang et al., 2018; Ovung et al., 2017). With the advancement of science and point analysing instruments, many minerals have been used for lithological and tectonic studies (Liang et al., 2018; Leterrier et al., 1982; Jacamon et al., 2009). Therefore, the use of chemical composition of minerals is a good indicator for the analysis of petrological processes. The study area is located in northern Iran in Guilan province between $49^{\circ} 55^{\prime}$ and $50^{\circ} 05^{\prime}$ eastern latitudes and $36^{\circ} 35^{\prime}$ and $36^{\circ} 45^{\prime}$ northern longitudes and is part of the Alborz mountain range. This mountain range itself is affected by Alpine-Himalayan orogeny. Its prominent characteristi is the abundance of platform sediments, which include limestone, dolomite and destrital rocks (Ghorbani, 2013). The study area contains a large volume of volcanic rocks (Eocene) and intrusive masses (Oligomyocene) in the structural zone of Western Alborz. Following compressive forces and stresses arising from the Middle and Late Alpine orogenic events, in this part of Alborz, a thick sequence of volcaniclastic and extrusive rocks (andesitic, basaltic and trachyandesitic) and intrusive masses (gabbroic, dioriteic, monzonitic) which intruded in these Eocene rocks and created alteration halos formed (Teimouri et al 2018). In the study area, intrusive masses (oligomiocene) are located next to Jirandeh fault and the main mass is in the form of an elongated batholith with a west-east trend at the outcrop surface. In this research, we seek to find out the origin of magma and their tectonomagmatic position according to the mineralogical compositions of the minerals forming the above intrusive masses.

\section{Geology}

Exposed rock units in the study area, from old to new, often include limestones of Mobarak formation (Carboniferous), clastic sediments of Doroud formation and calcareous rocks of Ruteh formation (Permian), mostly silisiclastic sediments of Shemshak formation (Jurassic) (Bahrfirozi et al., 2004), detrital sediments of Fajan formation (Paleocene), volcanic rocks (lava and pyroclasts) belonged to Karaj formation (Eocene) (Ghalamghash et al., 2002), and Oligomiocene intrusive masses respectively (Figure 1). Eocene volcanic rocks in the area include pyroclastic and outer igneous rocks. Extrusive rocks in this area include andesitic, basaltic and trachyandesitic types. Many intrusive masses have intruded into the Eocene volcanic-clastic assemblages, and therefore the age of these masses is post-Eocene (and probably belong to the Oligomiocene). Petrographic study of these intrusions shows that most of these rocks are basic and often gabbroic and some are monzoniteic. These rocks are usually seen in the form of small 
stocks with an outcrop of about a few tens of square meters (Figure 2). At the place of contact of these masses with the surrounding rocks, the fault zones or alteration zones can be seen.

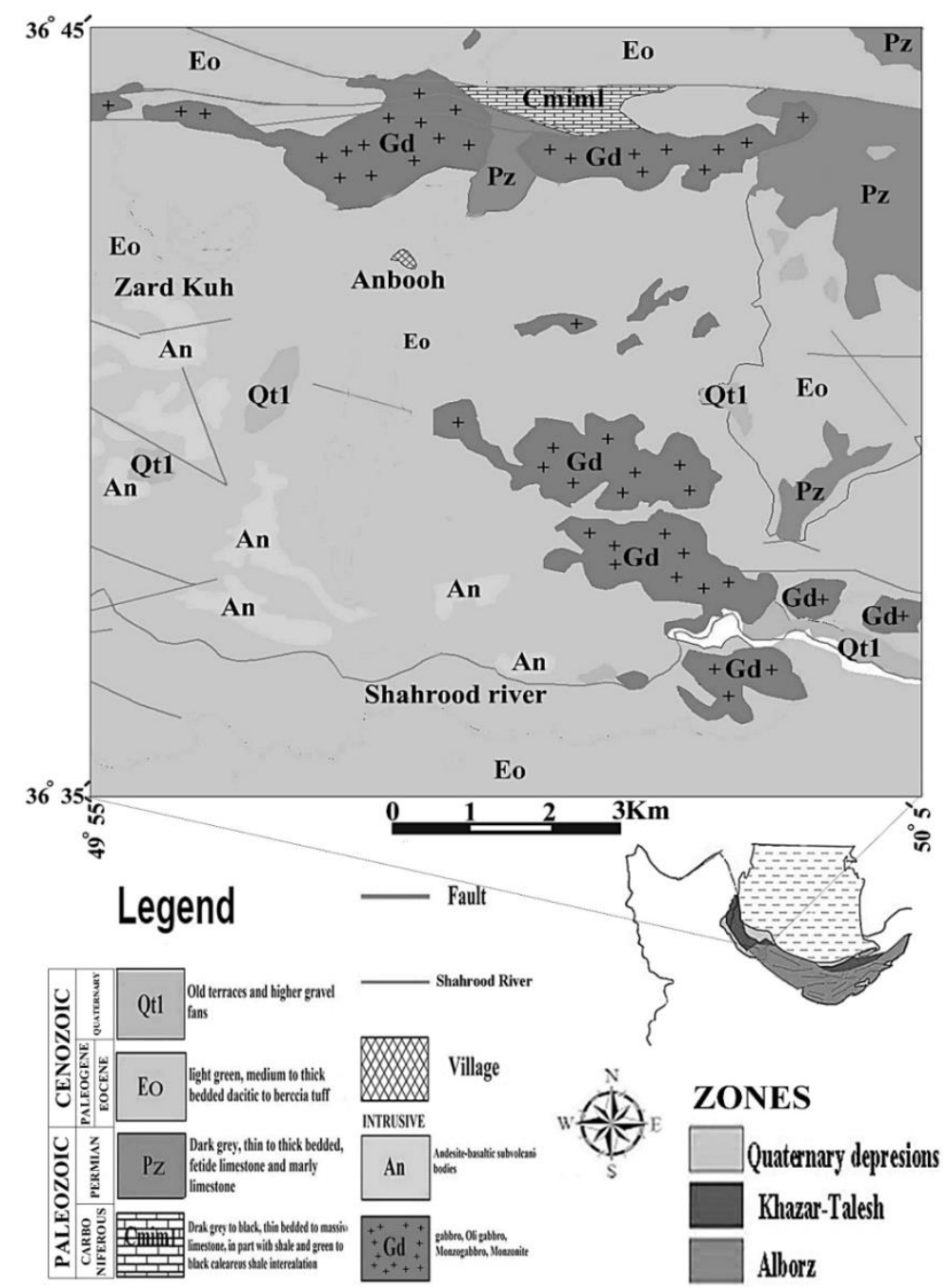

Figure 1. Simplified geological map (Ghalamghash et al., 2002 \& Baharfiruzi et al., 2003) and the location of the study area on it and the structural division map of Iran (Nogole-Sadat 1993). As can be seen, this area is located in Alborz.

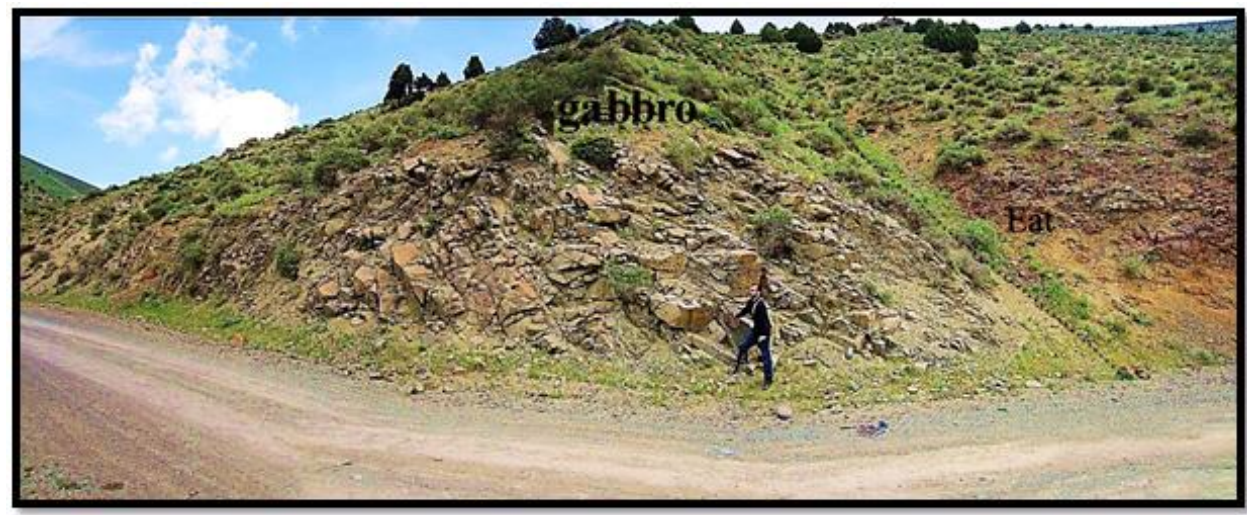

Figure 2. Exposure of intrusive rocks with gabbro composition located $4 \mathrm{~km}$ northwest of Anbouh village. 


\section{METHODOLOGY}

After sampling the intrusive masses of the study area and petrographical studies, in order to know the chemical compositions and their use in petrogenetic issues, the samples were sent to the Iranian Mineral Processing Research Center (IMPRC) in Karaj for spot chemical analysis of minerals by EPMA (electron microprobe microanalysis) method. In this center, first thin-polished sections were prepared from the samples, then after carbon coating, the samples were analysed using Cameca company SX100 microprocessor in laboratory conditions including accelerator voltage of $20 \mathrm{KV}$, current intensity of 10 $\mathrm{nA}$, temperature of $25^{\circ} \mathrm{C}$ and humidity of 30\%. Minpet 2.02 and Excel software were used to draw graphs and cationic calculations of the analysis results. The results of spot analysis of plagioclase, pyroxene, amphibole, olivine, biotite, magnetite and alkaline feldspars minerals are given in Table 1 to 7.

Table 1. Results of electron microprobe analysis of amphibole minerals and calculation of the number of cations based on 22 oxygens.

\begin{tabular}{|c|c|c|c|}
\hline \multirow{2}{*}{$\begin{array}{ll}\text { Analyte } & \text { Sample } \\
\end{array}$} & B20Am7 & B20Am8 & B20Am9 \\
\hline & Monzonite porphyry & Monzonite porphyry & Monzonite porphyry \\
\hline $\mathrm{SiO} 2$ & 43.32 & 42.81 & 42.27 \\
\hline $\mathrm{Al} 2 \mathrm{O} 3$ & 11.21 & 11.23 & 10.35 \\
\hline TiO2 & 3.2 & 3.35 & 3.4 \\
\hline $\mathrm{Cr} 2 \mathrm{O} 3$ & 0.03 & 0 & 0 \\
\hline $\mathrm{FeO}^{*}$ & 11.49 & 11.58 & 12.2 \\
\hline $\mathrm{MgO}$ & 13.16 & 13.37 & 13.53 \\
\hline $\mathrm{MnO}$ & 0.25 & 1.3 & 1.4 \\
\hline $\mathrm{K} 2 \mathrm{O}$ & 1.04 & 1.02 & 1.3 \\
\hline $\mathrm{CaO}$ & 11.75 & 11.61 & 11.67 \\
\hline $\mathrm{Na} 2 \mathrm{O}$ & 2.51 & 2.53 & 2.44 \\
\hline $\mathrm{Cl}$ & 0 & 0 & 0 \\
\hline $\mathrm{F}$ & 0 & 0 & 0 \\
\hline Total & 97.93 & 98.80 & 98.56 \\
\hline $\mathrm{O}-\mathrm{F}-\mathrm{Cl}$ & 0 & 0 & 0 \\
\hline $\mathrm{O}-\mathrm{F}$ & 0 & 0 & 0 \\
\hline $\mathrm{O}-\mathrm{Cl}$ & 0 & 0 & 0 \\
\hline $\mathrm{Si}$ & 6.403 & 6.274 & 6.238 \\
\hline $\mathrm{Al}$ & 1.597 & 1.726 & 1.758 \\
\hline $\mathrm{Fe} 3$ & 0 & 0 & 0.004 \\
\hline $\mathrm{Ti}$ & 0 & 0 & 0 \\
\hline Sum_T & 8 & 8 & 8 \\
\hline $\mathrm{Al}$ & 0.354 & 0.212 & 0.041 \\
\hline $\mathrm{Cr}$ & 0.004 & 0 & 0 \\
\hline $\mathrm{Fe} 3$ & 0 & 0.173 & 0.004 \\
\hline $\mathrm{Ti}$ & 0.356 & 0.369 & 0 \\
\hline $\mathrm{Mg}$ & 2.900 & 2.921 & 2.977 \\
\hline
\end{tabular}




\begin{tabular}{|c|c|c|c|}
\hline Fe2 & 1.371 & 1.245 & 1.284 \\
\hline $\mathrm{Mn}$ & 0.016 & 0.080 & 0.103 \\
\hline $\mathrm{Ca}$ & 0 & 0 & 0 \\
\hline Sum_C & 5 & 5 & 5 \\
\hline $\mathrm{Mg}$ & 0 & 0 & 0 \\
\hline $\mathrm{Fe} 2$ & 0.049 & 0.002 & 0.072 \\
\hline $\mathrm{Mn}$ & 0.016 & 0.081 & 1.845 \\
\hline $\mathrm{Ca}$ & 1.861 & 1.823 & 0.082 \\
\hline $\mathrm{Na}$ & 0.074 & 0.094 & 2 \\
\hline Sum_B & 2 & 2 & 0 \\
\hline $\mathrm{Ca}$ & 0 & 0 & \\
\hline
\end{tabular}

Table 2. Results of electron microprobe analysis of pyroxene minerals and calculation of the number of

\begin{tabular}{|c|c|c|c|c|c|c|c|c|c|c|}
\hline Samp & $\begin{array}{c}\text { B10P } \\
\times 38 \\
\end{array}$ & $\begin{array}{c}\text { B10P } \\
\times 39\end{array}$ & $\begin{array}{c}\text { B12Px1 } \\
8\end{array}$ & $\begin{array}{c}\text { B12Px } \\
19\end{array}$ & $\begin{array}{c}\text { B12PX } \\
20\end{array}$ & $\begin{array}{c}\text { B15P } \\
\times 12\end{array}$ & $\begin{array}{c}\text { B15P } \\
\text { x13 }\end{array}$ & $\begin{array}{c}\text { B15P } \\
\times 20\end{array}$ & $\begin{array}{c}\text { B15P } \\
\text { x21 }\end{array}$ & $\begin{array}{c}\text { B15P } \\
\times 22\end{array}$ \\
\hline $\begin{array}{r}\text { Analy } \\
\text { te }\end{array}$ & $\begin{array}{l}\text { Olivin } \\
\mathrm{e} \\
\text { gabbr } \\
\quad \mathrm{o}\end{array}$ & $\begin{array}{c}\text { Olivin } \\
\text { e } \\
\text { gabbr } \\
\text { o }\end{array}$ & $\begin{array}{c}\text { Hornfel } \\
\text { s }\end{array}$ & $\begin{array}{c}\text { Hornfel } \\
\text { s }\end{array}$ & $\begin{array}{c}\text { Hornfel } \\
\text { s }\end{array}$ & $\begin{array}{c}\text { Gabb } \\
\text { ro }\end{array}$ & $\begin{array}{c}\text { Gabb } \\
\text { ro }\end{array}$ & $\begin{array}{c}\text { Gabb } \\
\text { ro }\end{array}$ & $\begin{array}{c}\text { Gabb } \\
\text { ro }\end{array}$ & $\begin{array}{c}\text { Gabbr } \\
\text { o }\end{array}$ \\
\hline $\mathrm{SiO} 2$ & 50.75 & 50.97 & 50.75 & 50.56 & 44.86 & 51.01 & 54.87 & 2.37 & 51.01 & 50.55 \\
\hline $\begin{array}{c}\mathrm{A} 12 \mathrm{O} \\
3\end{array}$ & 0.81 & 0.74 & 1.53 & 1.23 & 5.5 & 1.68 & 1.46 & 0.86 & 0.71 & 0.62 \\
\hline $\mathrm{TiO} 2$ & 0.36 & 0.3 & 0.49 & 0.4 & 1.01 & 1.06 & 0.31 & 0.36 & 0.38 & 0.4 \\
\hline $\begin{array}{c}\mathrm{Cr} 2 \mathrm{O} \\
3\end{array}$ & 0.03 & 0.02 & 0.08 & 0.03 & 0 & 0.11 & 0.08 & 0.09 & 0.03 & 0 \\
\hline $\mathrm{FeO}^{*}$ & 21 & 20.79 & 9.32 & 9.34 & 14.5 & 12.33 & 13.12 & 20.83 & 22.72 & 26.28 \\
\hline $\mathrm{MgO}$ & 23.22 & 23.33 & 14.7 & 14.13 & 7.87 & 15.76 & 18.56 & 22.86 & 21.06 & 18.84 \\
\hline $\mathrm{MnO}$ & 0.49 & 0.62 & 0 & 0.71 & 0 & 0.32 & 0.27 & 0.49 & 0.44 & 0.61 \\
\hline $\mathrm{K} 2 \mathrm{O}$ & 0 & 0 & 0 & 0 & 0 & 0.1 & 0 & 0 & 0.03 & 0 \\
\hline $\mathrm{CaO}$ & 1.62 & 1.39 & 21.14 & 21.15 & 23.33 & 17.69 & 11.6 & 2.08 & 1.77 & 1.75 \\
\hline $\mathrm{Na} 2 \mathrm{O}$ & 0.15 & 0.03 & 0.58 & 0.8 & 0.7 & 0.3 & 0.15 & 0 & 0 & 0 \\
\hline Total & 98.54 & 98.19 & 98.59 & 98.35 & 97.99 & $\begin{array}{c}100.6 \\
7\end{array}$ & $\begin{array}{c}100.5 \\
7\end{array}$ & 99.94 & 98.15 & 99.05 \\
\hline $\mathrm{Si}$ & 1.927 & 1.936 & 1.928 & 1.934 & 1.788 & 1.912 & 2.003 & 1.951 & 1.955 & 1.954 \\
\hline $\mathrm{Ti}$ & 0.010 & 0.009 & 0.014 & 0.012 & 0.030 & 0.030 & 0.009 & 0.010 & 0.011 & 0.012 \\
\hline $\mathrm{Al}$ & 0.036 & 0.033 & 0.068 & 0.055 & 0.258 & 0.074 & 0.063 & 0.038 & 0.032 & 0.028 \\
\hline $\mathrm{Fe}(\mathrm{II})$ & 0.667 & 0.661 & 0.296 & 0.299 & 0.483 & 0.386 & 0.401 & 0.649 & 0.728 & 0.849 \\
\hline $\mathrm{Ca}$ & 0.066 & 0.057 & 0.860 & 0.867 & 0.997 & 0.710 & 0.454 & 0.083 & 0.073 & 0.072 \\
\hline
\end{tabular}




\begin{tabular}{|c|c|c|c|c|c|c|c|c|c|c|}
\hline $\mathrm{Na}$ & 0.011 & 0.002 & 0.043 & 0.059 & 0.054 & 0.022 & 0.011 & 0 & 0 & 0 \\
\hline $\mathrm{K}$ & 0 & 0 & 0 & 0 & 0 & 0.005 & 0 & 0 & 0.001 & 0 \\
\hline Total & 4.049 & 4.039 & 4.044 & 4.056 & 4.079 & 4.033 & 3.961 & 4.019 & 4.018 & 4.021 \\
\hline
\end{tabular}

Continuation of Table 2

\begin{tabular}{|c|c|c|c|c|c|c|c|c|}
\hline Sample & B20Px10 & B20Px11 & B20Px12 & B20Px13 & B20Px14 & B20Px15 & B20Px16 & B20Px17 \\
\cline { 2 - 9 } Analyte & $\begin{array}{c}\text { Monzonite } \\
\text { pophyry }\end{array}$ & $\begin{array}{c}\text { Monzonite } \\
\text { porphyry }\end{array}$ & $\begin{array}{c}\text { Monzonite } \\
\text { porphyry }\end{array}$ & $\begin{array}{c}\text { Monzonite } \\
\text { porphyry }\end{array}$ & $\begin{array}{c}\text { Monzonite } \\
\text { porphyry }\end{array}$ & $\begin{array}{c}\text { Monzonite } \\
\text { porphyry }\end{array}$ & $\begin{array}{c}\text { Monzonite } \\
\text { porphyry }\end{array}$ & $\begin{array}{c}\text { Monzonite } \\
\text { porphyry }\end{array}$ \\
\hline $\mathrm{SiO} 2$ & 50.39 & 50.61 & 50.68 & 50.1 & 49.58 & 51.62 & 49.76 & 50.59 \\
\hline $\mathrm{Al2O} 3$ & 3.02 & 2.75 & 3.13 & 2.7 & 3.69 & 1.77 & 2.55 & 1.95 \\
\hline $\mathrm{TiO} 2$ & 0.74 & 0.58 & 0.81 & 0.61 & 0.74 & 0.24 & 0.55 & 0.28 \\
\hline $\mathrm{Cr} 2 \mathrm{O} 3$ & 0 & 0 & 0 & 0 & 0 & 0 & 0.05 & 0 \\
\hline $\mathrm{FeO} *$ & 8.29 & 8.92 & 8.3 & 8.64 & 7.84 & 7.57 & 7.59 & 8.46 \\
\hline $\mathrm{MgO}$ & 15.47 & 15.64 & 15.54 & 15.55 & 14.93 & 15.19 & 15.09 & 14.63 \\
\hline $\mathrm{MnO}$ & 0.33 & 0 & 0.37 & 0.48 & 0 & 0 & 0 & 0.73 \\
\hline $\mathrm{K} 2 \mathrm{O}$ & 0 & 0 & 0 & 0 & 0 & 0 & 0.81 & 0 \\
\hline $\mathrm{CaO}$ & 21.32 & 21.15 & 20.83 & 21.11 & 21.26 & 22.22 & 21.8 & 21.71 \\
\hline $\mathrm{Na} 2 \mathrm{O}$ & 0.39 & 0.49 & 0.45 & 0.51 & 0.42 & 0.39 & 0.43 & 0.38 \\
\hline $\mathrm{Total}$ & 100.12 & 100.14 & 100.11 & 99.70 & 98.77 & 99 & 98.63 & 98.73 \\
\hline $\mathrm{Si}$ & 1.884 & 1.888 & 1.885 & 1.881 & 1.873 & 1.937 & 1.889 & 1.919 \\
\hline $\mathrm{Ti}$ & 0.021 & 0.016 & 0.023 & 0.017 & 0.021 & 0.007 & 0.016 & 0.008 \\
\hline $\mathrm{Al}$ & 0.133 & 0.121 & 0.137 & 0.119 & 0.164 & 0.078 & 0.114 & 0.087 \\
\hline $\mathrm{Fe}(\mathrm{II})$ & 0.259 & 0.278 & 0.258 & 0.271 & 0.248 & 0.238 & 0.241 & 0.268 \\
\hline $\mathrm{Ca}$ & 0.854 & 0.845 & 0.830 & 0.849 & 0.861 & 0.893 & 0.887 & 0.882 \\
\hline $\mathrm{Na}$ & 0.028 & 0.035 & 0.032 & 0.037 & 0.031 & 0.028 & 0.032 & 0.028 \\
\hline $\mathrm{K}$ & 0 & 0 & 0 & 0 & 0 & 0 & 0.039 & 0 \\
\hline $\mathrm{Total}$ & 4.043 & 4.053 & 4.040 & 4.061 & 4.039 & 4.031 & 4.073 & 4.043 \\
\hline
\end{tabular}

Table 3. Results of electron microprobe analysis of biotite minerals and calculation of cations based on 8 oxygens.

\begin{tabular}{|c|c|c|c|}
\hline \multirow{2}{*}{ Analyte Sample } & B20Bt1 & B20Bt2 & B20Bt3 \\
\cline { 2 - 4 } & Monzonite porphyry & Monzonite porphyry & Monzonite porphyry \\
\hline $\mathrm{SiO} 2$ & 36.73 & 35.54 & 35.91 \\
\hline $\mathrm{Al} 2 \mathrm{O} 3$ & 13.3 & 14.18 & 15.06 \\
\hline $\mathrm{TiO} 2$ & 4.15 & 4.52 & 4.08 \\
\hline $\mathrm{Cr} 2 \mathrm{O} 3$ & 0 & 0 & 0 \\
\hline $\mathrm{FeO}$ & 17.95 & 15.84 & 12.88 \\
\hline $\mathrm{MgO}$ & 15.82 & 16.05 & 0.15 \\
\hline $\mathrm{MnO}$ & 0.84 & 0.83 & 8.32 \\
\hline $\mathrm{K} 2 \mathrm{O}$ & 7.34 & 8.48 & 0.57 \\
\hline $\mathrm{CaO}$ & 0.29 & 0.34 & \\
\hline
\end{tabular}




\begin{tabular}{|c|c|c|c|}
\hline $\mathrm{Na} 2 \mathrm{O}$ & 0.35 & 0.2 & 0.34 \\
\hline Total & 96.77 & 95.98 & 95.74 \\
\hline $\mathrm{Si}$ & 2.647 & 2.663 & 2.647 \\
\hline $\mathrm{Ti}$ & 0.225 & 0.255 & 0.226 \\
\hline $\mathrm{Al}$ & 1.130 & 1.252 & 1.309 \\
\hline $\mathrm{Fe}(\mathrm{II})$ & 1.082 & 0.993 & 0.794 \\
\hline $\mathrm{Ca}$ & 0.022 & 0.027 & 0.045 \\
\hline $\mathrm{Na}$ & 0.049 & 0.029 & 0.049 \\
\hline $\mathrm{K}$ & 0.675 & 0.811 & 0.783 \\
\hline Total & 7.925 & 7.876 & 7.888 \\
\hline
\end{tabular}

Table 4. Results of electron microprobe analysis of plagioclase minerals and calculation of cations based

\begin{tabular}{|c|c|c|c|c|c|c|c|}
\hline \multirow{2}{*}{ Anample } & B10P141 & B10PI42 & B10P143 & B10PI50 & B10Pl51 & B10PI52 & B10PI53 \\
\cline { 2 - 8 } & $\begin{array}{c}\text { Olivine } \\
\text { gabbro }\end{array}$ & $\begin{array}{c}\text { Olivine } \\
\text { gabbro }\end{array}$ & $\begin{array}{c}\text { Olivine } \\
\text { gabbro }\end{array}$ & $\begin{array}{c}\text { Olivine } \\
\text { gabbro }\end{array}$ & $\begin{array}{c}\text { Olivine } \\
\text { gabbro }\end{array}$ & $\begin{array}{c}\text { Olivine } \\
\text { gabbro }\end{array}$ & $\begin{array}{c}\text { Olivine } \\
\text { gabbro }\end{array}$ \\
\hline $\mathrm{SiO} 2$ & 55.99 & 53.29 & 53.83 & 55 & 55.53 & 54.25 & 55.42 \\
\hline $\mathrm{Al} 2 \mathrm{O} 3$ & 24.58 & 26.73 & 24.77 & 25.43 & 26.64 & 26.95 & 25.1 \\
\hline $\mathrm{TiO} 2$ & 0.04 & 0 & 24.77 & 0.07 & 0.05 & 0.08 & 0.02 \\
\hline $\mathrm{FeO} *$ & 0.52 & 0.34 & 0.56 & 0.46 & 0.31 & 0.38 & 0.5 \\
\hline $\mathrm{MgO}$ & 0.04 & 0.02 & 0.02 & 0.04 & 0 & 0.03 & 0.04 \\
\hline $\mathrm{MnO}$ & 0.04 & 0.04 & 0.06 & 0 & 0 & 0 & 0 \\
\hline $\mathrm{K} 2 \mathrm{O}$ & 0.57 & 0.2 & 0.48 & 0.28 & 0.33 & 0.17 & 0.48 \\
\hline $\mathrm{CaO}$ & 10.37 & 14.63 & 12.43 & 11.72 & 11.73 & 13.21 & 10.81 \\
\hline $\mathrm{Na} 2 \mathrm{O}$ & 6.81 & 4.67 & 5.95 & 5.49 & 4.4 & 4.42 & 6.16 \\
\hline $\mathrm{Total}$ & 98.96 & 99.92 & 98.19 & 98.49 & 98.99 & 99.49 & 98.53 \\
\hline $\mathrm{Si}$ & 2.573 & 2.443 & 2.510 & 2.535 & 2.531 & 2.478 & 2.553 \\
\hline $\mathrm{Ti}$ & 0.001 & 0 & 0.003 & 0.002 & 0.002 & 0.003 & 0.001 \\
\hline $\mathrm{Al}$ & 1.331 & 1.444 & 1.361 & 1.381 & 1.431 & 1.451 & 1.363 \\
\hline $\mathrm{Fe}(\mathrm{II})$ & 0.020 & 0.013 & 0.022 & 0.018 & 0.012 & 0.015 & 0.019 \\
\hline $\mathrm{Ca}$ & 0.511 & 0.718 & 0.621 & 0.579 & 0.573 & 0.646 & 0.534 \\
\hline $\mathrm{Na}$ & 0.607 & 0.415 & 0.538 & 0.491 & 0.389 & 0.391 & 0.550 \\
\hline $\mathrm{K}$ & 0.033 & 0.012 & 0.029 & 0.016 & 0.019 & 0.010 & 0.028 \\
\hline $\mathrm{Total}$ & 5.080 & 5.048 & 5.089 & 5.025 & 4.956 & 4.995 & 5.053 \\
\hline
\end{tabular}

Continuation of Table 4

\begin{tabular}{|c|c|c|c|c|c|c|}
\hline \multirow{2}{*}{ Sample } & B12P126 & B12PI27 & B12PI34 & B12P135 & B10P154 & B10P55 \\
\cline { 2 - 7 } & Hornfels & Hornfels & Hornfels & Hornfels & Olivine gabbro & Olivine gabbro \\
\hline $\mathrm{SiO} 2$ & 66.12 & 65.61 & 35.79 & 37.13 & 54.01 & 55.58 \\
\hline $\mathrm{Al2O} 3$ & 18.95 & 20.85 & 28.77 & 29.67 & 26.4 & 24.59 \\
\hline $\mathrm{TiO} 2$ & 0 & 0.08 & 0.05 & 0.11 & 0.09 & 0.06 \\
\hline $\mathrm{FeO} *$ & 0.01 & 0.09 & 0.23 & 0.12 & 0.68 & 0.51 \\
\hline $\mathrm{MgO}$ & 0 & 0 & 0 & 0.01 & 0.27 & 0.04 \\
\hline $\mathrm{MnO}$ & 0.06 & 0 & 0 & 0.02 & 0.03 & 0.03 \\
\hline $\mathrm{K} 2 \mathrm{O}$ & 1.57 & 0.01 & 12.26 & 10.46 & 0.16 & 0.81 \\
\hline
\end{tabular}




\begin{tabular}{|c|c|c|c|c|c|c|}
\hline $\mathrm{CaO}$ & 0.63 & 1.53 & 20.35 & 19.91 & 13.88 & 9.96 \\
\hline $\mathrm{Na} 2 \mathrm{O}$ & 11.98 & 10.39 & 0 & 0 & 3.77 & 6.56 \\
\hline $\mathrm{Total}$ & 99.32 & 98.56 & 97.45 & 97.43 & 99.29 & 98.14 \\
\hline $\mathrm{Si}$ & 2.954 & 2.916 & 1.880 & 1.916 & 2.476 & 2.573 \\
\hline $\mathrm{Ti}$ & 0 & 0.003 & 0.002 & 0.004 & 0.003 & 0.002 \\
\hline $\mathrm{Al}$ & 0.998 & 1.092 & 1.781 & 1.805 & 1.427 & 1.342 \\
\hline $\mathrm{Fe}(\mathrm{II})$ & 0 & 0.003 & 0.010 & 0.005 & 0.026 & 0.020 \\
\hline $\mathrm{Ca}$ & 0.030 & 0.073 & 1.145 & 1.101 & 0.682 & 0.494 \\
\hline $\mathrm{Na}$ & 1.038 & 0.895 & 0 & 0 & 0.335 & 0.589 \\
\hline $\mathrm{K}$ & 0.089 & 0.001 & 0.821 & 0.689 & 0.009 & 0.048 \\
\hline $\mathrm{Total}$ & 5.111 & 4.983 & 5.639 & 5.522 & 4.979 & 5.072 \\
\hline
\end{tabular}

Continuation of Table 4

\begin{tabular}{|c|c|c|c|c|c|c|c|c|}
\hline Sample & B15PI1 & B15PI2 & B15PI3 & B15PI4 & B15PI5 & B15PI24 & B15Pl25 & B15PI26 \\
\hline Analyte & Gabbro & Gabbro & Gabbro & Gabbro & Gabbro & Gabbro & Gabbro & Gabbro \\
\hline $\mathrm{SiO} 2$ & 53.73 & 53.65 & 52.53 & 53.86 & 50.92 & 51.86 & 53.98 & 56.75 \\
\hline $\mathrm{Al} 2 \mathrm{O} 3$ & 27.62 & 27.78 & 28.62 & 27.04 & 21.27 & 28.54 & 26.73 & 25.71 \\
\hline $\mathrm{TiO} 2$ & 0.05 & 0.34 & 0.13 & 0.04 & 0.07 & 0 & 0.04 & 0.05 \\
\hline $\mathrm{FeO}^{*}$ & 0.37 & 0.37 & 0.41 & 0.47 & 4.6 & 0.46 & 0.42 & 0.41 \\
\hline $\mathrm{MgO}$ & 0 & 0 & 0 & 0.02 & 1.84 & 0.04 & 0.02 & 0.02 \\
\hline $\mathrm{MnO}$ & 0 & 0 & 0.04 & 0 & 0.11 & 0.09 & 0 & 0.07 \\
\hline $\mathrm{K} 2 \mathrm{O}$ & 0.46 & 0.2 & 0.44 & 0.6 & 0.24 & 0.26 & 0.37 & 0.23 \\
\hline $\mathrm{CaO}$ & 11.38 & 11.23 & 11.94 & 10.77 & 4.85 & 13.64 & 11.44 & 8.21 \\
\hline $\mathrm{Na} 2 \mathrm{O}$ & 5.16 & 4.51 & 4.96 & 5.67 & 5.61 & 4.66 & 5.14 & 6.85 \\
\hline Total & 98.77 & 98.08 & 99.07 & 98.47 & 89.51 & 99.55 & 98.14 & 98.30 \\
\hline $\mathrm{Si}$ & 2.646 & 2.469 & 2.414 & 2.483 & 2.594 & 2.384 & 2.493 & 2.593 \\
\hline $\mathrm{Ti}$ & 0.002 & 0.012 & 0.004 & 0.001 & 0.003 & 0 & 0.001 & 0.002 \\
\hline $\mathrm{Al}$ & 1.603 & 1.507 & 1.550 & 1.469 & 1.277 & 1.547 & 1.455 & 1.385 \\
\hline $\mathrm{Fe}(\mathrm{II})$ & 0.015 & 0.014 & 0.016 & 0.018 & 0.196 & 0.018 & 0.016 & 0.016 \\
\hline $\mathrm{Ca}$ & 0.020 & 0.554 & 0.588 & 0.532 & 0.265 & 0.672 & 0.566 & 0.402 \\
\hline $\mathrm{Na}$ & 0.493 & 0.402 & 0.442 & 0.507 & 0.554 & 0.415 & 0.460 & 0.607 \\
\hline $\mathrm{K}$ & 0.029 & 0.012 & 0.026 & 0.035 & 0.016 & 0.015 & 0.022 & 0.013 \\
\hline Total & 4.810 & 4.972 & 5.041 & 5.050 & 5.049 & 5.058 & 5.018 & 5.022 \\
\hline
\end{tabular}

Continuation of Table 4

\begin{tabular}{|c|c|c|c|}
\hline \multirow{2}{*}{ Sample } & B20P14 & B20P15 & B20Pl6 \\
\cline { 2 - 4 } & Monzonite porphyry & Monzonite porphyry & Monzonite porphyry \\
\hline $\mathrm{SiO} 2$ & 54.09 & 60.2 & 63.16 \\
\hline $\mathrm{Al} 2 \mathrm{O} 3$ & 27.22 & 24.75 & 21.29 \\
\hline $\mathrm{TiO} 2$ & 0.13 & 0 & 0.05 \\
\hline $\mathrm{FeO} *$ & 0.22 & 0.12 & 0.33 \\
\hline $\mathrm{MgO}$ & 0.01 & 0.03 & 0 \\
\hline $\mathrm{MnO}$ & 1.98 & 0.57 & 2.03 \\
\hline
\end{tabular}




\begin{tabular}{|c|c|c|c|}
\hline $\mathrm{K} 2 \mathrm{O}$ & 9.76 & 0.68 & 0.66 \\
\hline $\mathrm{CaO}$ & 0.21 & 5.13 & 2.98 \\
\hline $\mathrm{Na} 2 \mathrm{O}$ & 6.39 & 8.14 & 9.39 \\
\hline $\mathrm{Total}$ & 100.01 & 99.62 & 99.89 \\
\hline $\mathrm{Si}$ & 2.470 & 2.696 & 2.827 \\
\hline $\mathrm{Ti}$ & 0.004 & 0 & 0.002 \\
\hline $\mathrm{Al}$ & 1.465 & 1.307 & 1.123 \\
\hline $\mathrm{Fe}(\mathrm{II})$ & 0.008 & 0.004 & 0.012 \\
\hline $\mathrm{Ca}$ & 0.478 & 0.246 & 0.143 \\
\hline $\mathrm{Na}$ & 0.566 & 0.707 & 0.815 \\
\hline $\mathrm{K}$ & 0.012 & 0.039 & 0.038 \\
\hline $\mathrm{Total}$ & 5.082 & 5.023 & 5.036 \\
\hline
\end{tabular}

Table 5. Results of electron microprobe analysis of alkali feldspars and calculation of cations based on 8

\begin{tabular}{|c|c|c|c|c|c|}
\hline \multirow{2}{*}{ Sample } & B15Afs 6 & B15Afs 7 & B15Afs 8 & B10Or44 & B10Or45 \\
\cline { 2 - 6 } Analyte & gabbro & \multirow{2}{*}{ gabbro } & gabbro & Olivine gabbro & Olivine gabbro \\
\hline $\mathrm{SiO} 2$ & 62.57 & 63.84 & 63.78 & 63.68 & 63.46 \\
\hline $\mathrm{Al2O} 3$ & 16.98 & 18.48 & 16.33 & 16.79 & 16.6 \\
\hline $\mathrm{TiO} 2$ & 1.33 & 0.07 & 0.39 & 0.06 & 0.05 \\
\hline $\mathrm{FeO} *$ & 0.08 & 0.05 & 0.17 & 0.44 & 0.32 \\
\hline $\mathrm{MgO}$ & 0 & 0 & 0 & 0.01 & 0 \\
\hline $\mathrm{MnO}$ & 0.01 & 0 & 0 & 0.01 & 0.01 \\
\hline $\mathrm{K} 2 \mathrm{O}$ & 17.01 & 0.92 & 16.88 & 15.3 & 0.21 \\
\hline $\mathrm{CaO}$ & 0 & 0.01 & 0.13 & 0.47 & 1.54 \\
\hline $\mathrm{Na} 2 \mathrm{O}$ & 0.85 & 0.92 & 1.6 & 1.79 & 98.52 \\
\hline $\mathrm{Total}$ & 98.83 & 100.46 & 99.28 & 98.61 & 3.008 \\
\hline $\mathrm{Si}$ & 2.963 & 2.964 & 3.008 & 3.004 & 0.002 \\
\hline $\mathrm{Ti}$ & 0.047 & 0.002 & 0.014 & 0.002 & 0.927 \\
\hline $\mathrm{Al}$ & 0.948 & 1.011 & 0.908 & 0.933 & 0.013 \\
\hline $\mathrm{Fe}(\mathrm{II})$ & 0.003 & 0.002 & 0.007 & 0.017 & 0.142 \\
\hline $\mathrm{Ca}$ & 0 & 0 & 0.007 & 0.024 & 0.988 \\
\hline $\mathrm{Na}$ & 0.078 & 0.083 & 0.146 & 0.164 & 5.091 \\
\hline $\mathrm{K}$ & 1.028 & 1.012 & 1.016 & 0.921 & 5.068 \\
\hline $\mathrm{Total}$ & 5.068 & 5.075 & 5.105 & & \\
\hline
\end{tabular}

Table 6. Results of electron microprobe analysis of oxide minerals of intrusive rocks in the study area.

\begin{tabular}{|c|c|c|c|}
\hline \multirow{2}{*}{ Analyte } & B15Mag 14 & B15Mag 15 & B15Mag 16 \\
\cline { 2 - 4 } & gabbro & gabbro & gabbro \\
\hline $\mathrm{Al2O} 3$ & 0.04 & 0.15 & 0.46 \\
\hline $\mathrm{Cr} 2 \mathrm{O} 3$ & 0.07 & 0.08 & 0.17 \\
\hline $\mathrm{TiO} 2$ & 46.56 & 45.61 & 2.75 \\
\hline $\mathrm{FeO} *$ & 49.62 & 48.47 & 46 \\
\hline $\mathrm{MgO}$ & 1.05 & 0.89 & 0.09 \\
\hline $\mathrm{MnO}$ & 0.75 & 1.57 & 07 \\
\hline
\end{tabular}




\begin{tabular}{|c|c|c|c|}
\hline $\mathrm{NaiO}$ & 0 & 0.66 & 0 \\
\hline $\mathrm{ZnO}$ & 0.43 & 0.43 & 0.43 \\
\hline $\mathrm{Total}$ & 100.7 & 100.24 & 92.75 \\
\hline $\mathrm{Ti}$ & 1.225 & 1.214 & 0.181 \\
\hline $\mathrm{Al}$ & 0.002 & 0.006 & 0.048 \\
\hline $\mathrm{Fe}(\mathrm{II})$ & 1.451 & 1.435 & 1.435 \\
\hline $\mathrm{Mn}$ & 0.022 & 0.047 & 0.007 \\
\hline $\mathrm{Ni}$ & 0.006 & 0.019 & 0.140 \\
\hline $\mathrm{Mg}$ & 0.055 & 0.047 & 0.028 \\
\hline $\mathrm{Zn}$ & 0.011 & 0.011 & 3.789 \\
\hline $\mathrm{Total}$ & 2.774 & 2.781 & \\
\hline
\end{tabular}

Table 7. Results of electron microprobe analysis of olivines and calculation of cations based on 4 oxygens.

\begin{tabular}{|c|c|c|c|c|c|}
\hline Sample & B100I 32 & B1001 33 & B1001 34 & B1001 35 & B1001 36 \\
\hline Analyte & $\begin{array}{l}\text { Olivine } \\
\text { gabbro }\end{array}$ & $\begin{array}{l}\text { Olivine } \\
\text { gabbro }\end{array}$ & $\begin{array}{l}\text { Olivine } \\
\text { gabbro }\end{array}$ & $\begin{array}{l}\text { Olivine } \\
\text { gabbro }\end{array}$ & $\begin{array}{l}\text { Olivine } \\
\text { gabbro }\end{array}$ \\
\hline $\mathrm{SiO} 2$ & 34.54 & 34.52 & 35.26 & 34.85 & 34.76 \\
\hline $\mathrm{Al} 2 \mathrm{O} 3$ & 0 & 0 & 0 & 0.04 & 0 \\
\hline $\mathrm{TiO} 2$ & 0.05 & 0.06 & 0.07 & 0.03 & 0.08 \\
\hline $\mathrm{FeO}^{*}$ & 30.86 & 31.6 & 31.79 & 32.6 & 32.98 \\
\hline $\mathrm{MgO}$ & 31.97 & 0.63 & 32.14 & 30.55 & 29.63 \\
\hline $\mathrm{MnO}$ & 0.71 & 0.63 & 0.67 & 0.63 & 0.71 \\
\hline $\mathrm{CaO}$ & 0 & 0.05 & 0.03 & 0.04 & 0.05 \\
\hline $\mathrm{K} 2 \mathrm{O}$ & 0 & 0 & 0 & 0 & 0 \\
\hline $\mathrm{NaiO}$ & 0 & 0.14 & 0.12 & 0.3 & 0 \\
\hline $\mathrm{Na} 2 \mathrm{O}$ & 0.02 & 0.02 & 0.08 & 0 & 0 \\
\hline Total & 98.24 & 98.92 & 100.16 & 99.04 & 98.21 \\
\hline $\mathrm{Si}$ & 0.999 & 1 & 1 & 1 & 1 \\
\hline $\mathrm{Ti}$ & 0.001 & 0.001 & 0.001 & 0.001 & 0.002 \\
\hline $\mathrm{Al}$ & 0 & 0 & 0 & 0.001 & 0 \\
\hline $\mathrm{Fe}(\mathrm{II})$ & 0.747 & 0.766 & 0.754 & 0.782 & 0.793 \\
\hline $\mathrm{Mn}$ & 0.017 & 0.015 & 0.016 & 0.015 & 0.017 \\
\hline $\mathrm{Na}$ & 0.001 & 0.001 & 0.004 & 0.001 & 0 \\
\hline $\mathrm{Mg}$ & 1.379 & 1.378 & 1.358 & 1.307 & 1.271 \\
\hline $\mathrm{Ca}$ & 0 & 0.002 & 0.001 & 0.001 & 0.002 \\
\hline $\mathrm{K}$ & 0 & 0 & 0 & 0 & 0 \\
\hline $\mathrm{Ni}$ & 0 & 0.003 & 0.003 & 0.007 & 0 \\
\hline
\end{tabular}

\section{PETROGRAPHY}

The intrusive rocks of the study area are mostly gabbro in terms of mineralogical composition, but intermediate types such as monzonite are also rarely seen. At the same time, sometimes there are enclaves of carbonate metamorphic rocks (calcsilicate hornfels) inside them. Petrographic studies show that monzonites have a porphyry texture with a relatively fine-grained groundmass (Figure 3) and phenocrysts according to their abundances are in plagioclase, amphibole, biotite, and opaque minerals, respectively. Plagioclases show little alteration. These minerals have obvious polysynthetic twins and often with very 
evident zoning. Their size varies from about 0.2 to about $1 \mathrm{~mm}$. In the rock, amphiboles with intersecting cleavages and brown to colorless pleochroism can be seen. Amphiboles are present only as phenocrystals and are between 0.6 and $2.0 \mathrm{~mm}$ in size. Biotite can also be seen in theses rocks such that some of them are fresh but others are completely altered to chlorite assemblages and other secondary minerals. The opaque mineral is found sparsely and in the form of phenocrysts in small amounts in the rock. The main part of the rock matrix is composed of entirely altered feldspars (orthoclases) and some quartz and opaque minerals.

The texture of the gabbros is often subhedral granular. Most gabbros do not have enclaves, but in some of them enclaves are rarely seen. The essential minerals are plagioclase and pyroxene (Figure 4) and the characterizing and minor accessory minerals found in various gabbro specimens include biotite, orthoclase, opaque, olivine (bolingite), zircon, sphene, and apatite. Latter minerals with low percentages are dispersed individually or in groups in different gabbro and are not found together in all samples. In some specimens, the amount of olivine is relatively high, so they can also be called olivine gabbro. Inside the intrusive bodies of the study area in the northeast of the Anbooh village, enclaves of calcsilicate hornfels can be seen. Petrographic studies show that these are mineral assemblages composed of metamorphic and secondary minerals. The texture of these rock is completely heterogeneous, so that parts of it are completely fine and consist of micro-cryptocrystalline minerals and other parts are granoblastic or porphyroblastic and contain a mixture of minerals such as calcite, garnet, plagioclase, clinopyroxene, sphene, leucoxene sphene, and wolastonite, epidote, zoisite and chlorite (Figure 5).

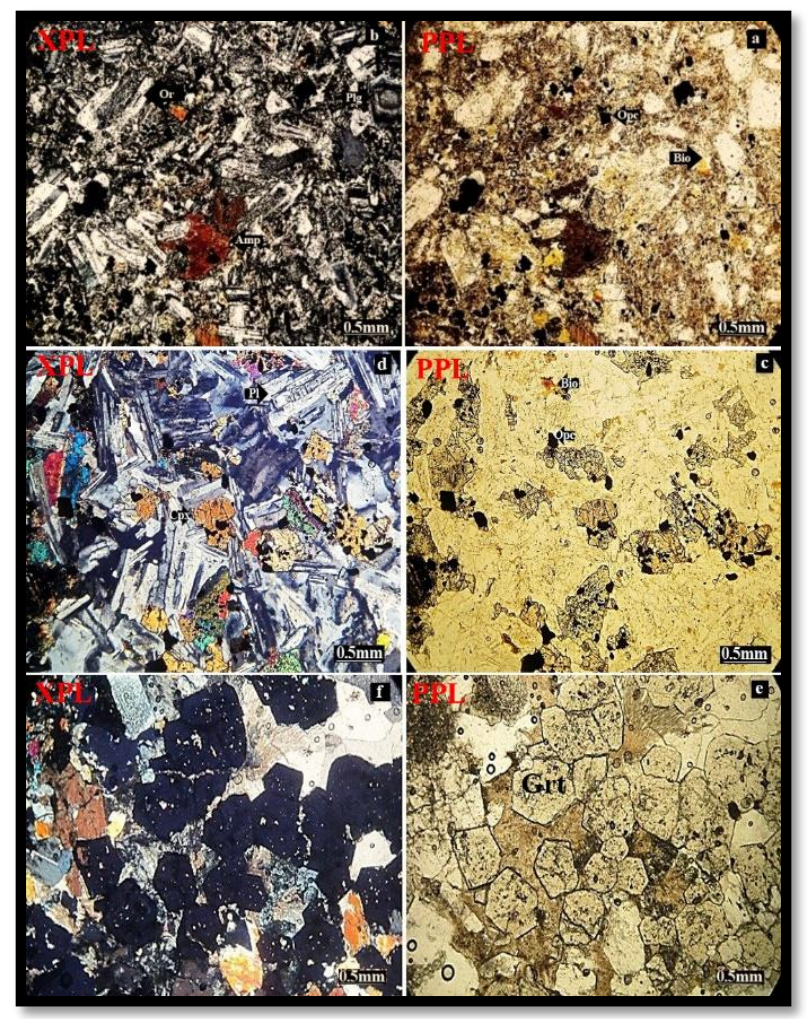

Figure 3. Microscopic images of: a, b) Monzonite with porphyry texture. Plagioclase, amphibole, biotite, and opaque minerals can be seen in the field of view. c, d) Gabbro with subhedral granular texture. Pyroxene, plagioclase and opaque minerals are seen in the microscopic field of view. e, f) Calcsilicate hornfels. Automorphic grossular minerals along with calcite, wollastonite, hedenbergite and diopside are seen in the field of view. Abreviations are: Grt: Garnet, Bio: Biotite, Or: Orthoclase, Plg: Plagioclase, Amp: Amphibole, Opc: Opaque, Cpx: Clinopyroxene. Right images are in ppl and left images are in xpl views. 


\section{DISCUSSION AND RESULTS}

\section{Chemistry of minerals \\ Amphibole}

For nomenclature of amphiboles, the report provided by the Subcommittee of the International Association of Mineralogy and the Commission on New Minerals and Mineral Names has been used. The general formula of amphibole is as follows.

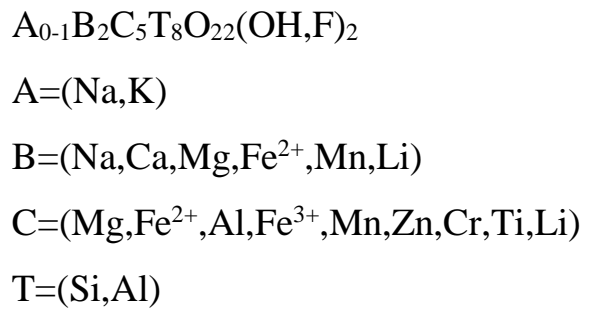

$\mathrm{A}_{0-1} \mathrm{~B}_{2} \mathrm{C}_{5} \mathrm{~T}_{8} \mathrm{O}_{22}(\mathrm{OH}, \mathrm{F})_{2}$

$\mathrm{A}=(\mathrm{Na}, \mathrm{K})$

$\mathrm{B}=\left(\mathrm{Na}, \mathrm{Ca}, \mathrm{Mg}, \mathrm{Fe}^{2+}, \mathrm{Mn}, \mathrm{Li}\right)$

$\mathrm{C}=\left(\mathrm{Mg}, \mathrm{Fe}^{2+}, \mathrm{Al}, \mathrm{Fe}^{3+}, \mathrm{Mn}, \mathrm{Zn}, \mathrm{Cr}, \mathrm{Ti}, \mathrm{Li}\right)$

$\mathrm{T}=(\mathrm{Si}, \mathrm{Al})$

The results of point analysis of amphiboles and calculation of cations based on 22 oxygen are given in Table 1. Based on the diagram BNa vs. BCa $+\mathrm{BNa}$ (Hawthorne, 1981), amphiboles are divided into four groups (Figure 6-a). The data obtained from the point analysis of the amphiboles of the selected specimens indicate that according to the classification (Leake et al., 1997), all these amphiboles are in the group of calcium amphiboles (Figure 4-a) and in the subgroup of hornblende pargasite and ferropargasite (ferrohornblende). At the same time, based on the results of microprobe analysis these amphiboles have zoning so that their core composition is (Table 1) ferropargazite (ferrohornblende), but middle and marginal parts, are pargasite (hornblende) ( Figure 4-b).
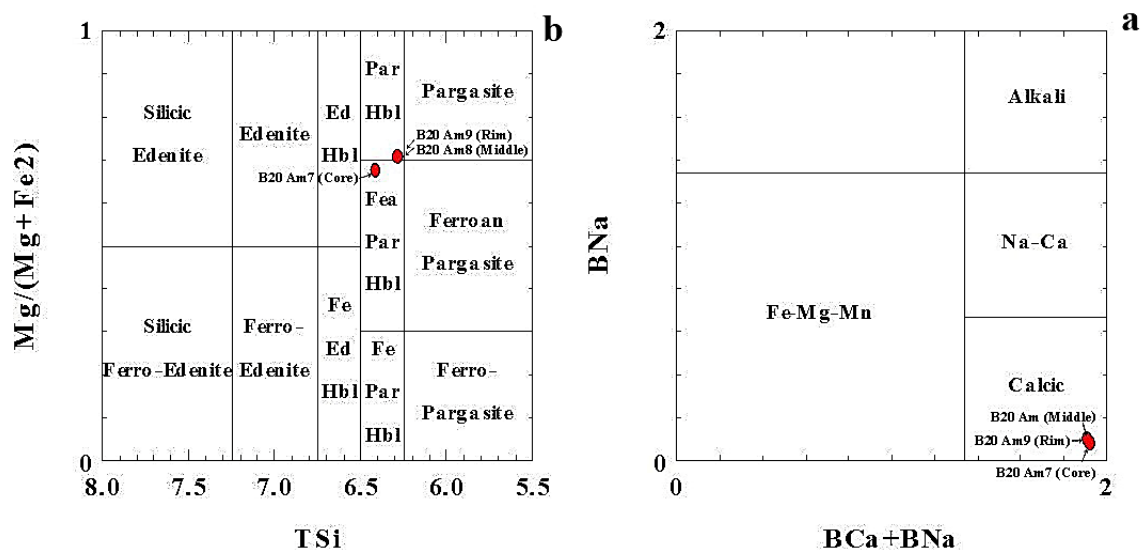

Figure 4. Chemical composition of amphiboles in the study area in the classification diagrams: a) General amphiboles (Hawthorne, 1981) b) Classification of calasic amphiboles (Leake et al., 1997), as shown in the diagrams, the amphiboles of the study area are of the calasic type and their chemical compositions are pargasite and ferropargasite.

\section{Pyroxene}

Pyroxenes are a member of inosilicates group, and in their structure, the $\mathrm{SiO} 4$ tetrahedrons are joined together by oxygen sharing to form a chain of tetrahedrons. The general formula of pyroxenes is as follows:

$\mathrm{B}_{1-\mathrm{P}} \mathrm{C}_{1+\mathrm{P}} \mathrm{T}_{2} \mathrm{O}_{6}$

$\mathrm{B}=(\mathrm{Na}, \mathrm{Ca})$ 
$\mathrm{C}=\left(\mathrm{Mg}, \mathrm{Fe}^{2+}, \mathrm{Al}, \mathrm{Fe}^{3+}, \mathrm{Mn}, \mathrm{Ni}, \mathrm{Cr}, \mathrm{Ti}, \mathrm{Li}\right)$

$\mathrm{T}=(\mathrm{Si}, \mathrm{Al})$

In the mentioned formula, the value of $\mathrm{P}$ in the series of orthopyroxenes is equal to one and its value in the series of monoclinic pyroxenes is set to zero. The calculation of cation values is given in (Table 2).

Based on $\mathbf{J}$ and $\mathrm{Q}$ indices (Morimoto, 1988) pyroxenes are divided into 4 groups: 1) Ca-Mg-Fe pyroxenes (Quad), 2) Ca-Na pyroxenes, 3) Na pyroxenes and 4) Other pyroxenes (Other) (Figure 5-a). The studied pyroxenes in the $\mathrm{J}-\mathrm{Q}$ diagram are in the field of iron-magnesium-calcium. In this diagram, the $\mathrm{J}$ and $\mathrm{Q}$ indices are obtained according to the following relation: $\mathrm{Q}=\mathrm{Ca}+\mathrm{Mg}+\mathrm{Fe}^{2+}$ and $\mathrm{J}=2 \mathrm{Na} \pm \mathrm{R}+\left(\mathrm{R}: \mathrm{Al}, \mathrm{Fe}^{3+}\right.$ $, \mathrm{Cr}^{3+}, \mathrm{Sc}^{3+}$ ). The triangle diagram En -Wo - Fs (Poldervaart \& Hess 1968) has also been used to name the pyroxenes present in the rocks of the study area (Figure 5-b, b1 to b4). The position of the microprobe data related to the pyroxenes of the samples studied on this diagram shows that most of them are clinopyroxene and have a composition of augite and diopside, while rarely some are orthopyroxene and are of the hypersthene type. The resulting data show that the composition of augites is such that their margins have more magnesium while their core have more iron and calcium. But hypersthenes have a more magnesic core and a ferric margin.
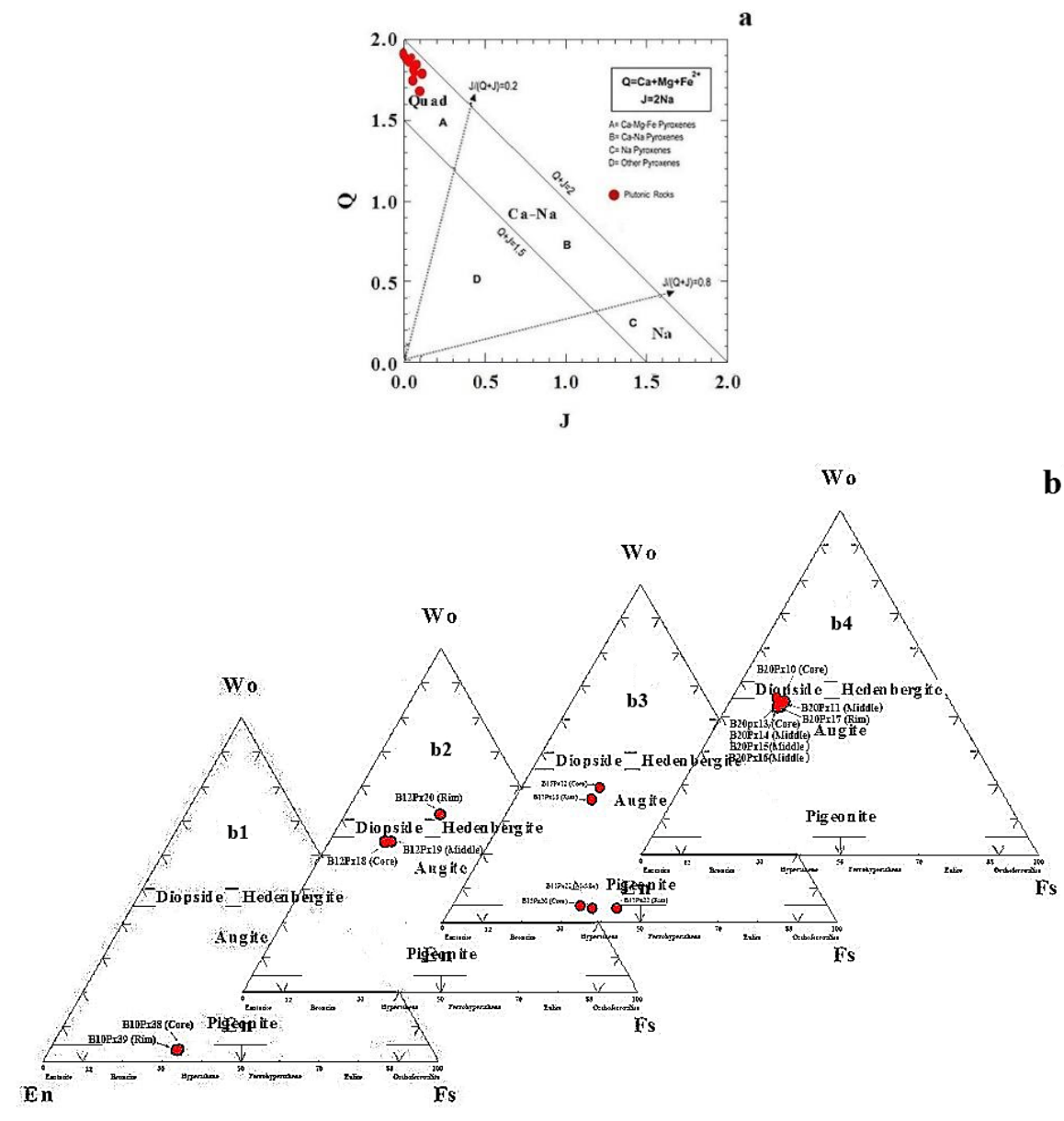

Figure 5. The plot of pyroxenes of the study area on: a) Q-J diagram (Morimoto, 1988), all of which, without exception, are in the realm of calcium-magnesium-iron pyroxenes. b) Triangle diagrams En -Wo - Fs (Poldervaart,1968). The b1 to b4 triangles refer to pyroxenes of olivine gabbro, calcsilicate hornfels, gabbro and monzonite porphyry respectively. As can be seen, the compositions of pyroxenes are in the fields of augite, diopside and hypersthene. 


\section{Biotite}

Biotite is an important ferromagnesian mineral in these rocks. Using the chemical composition of biotite, the physicochemical conditions of its formation can be detected (Deer et al., 1992). The general formula of mica is as follows:

$\mathrm{B}_{2} \mathrm{C}_{4-6} \mathrm{~T}_{8} \mathrm{O}_{20}(\mathrm{OH}, \mathrm{F})_{4}$

$\mathrm{B}=(\mathrm{K}, \mathrm{Na}, \mathrm{Ca}, \mathrm{Ba}, \mathrm{Rb}, \mathrm{Cs})$

$\mathrm{C}=\left(\mathrm{Al}^{6+}, \mathrm{Mg}, \mathrm{Fe}, \mathrm{Mn}, \mathrm{Cr}, \mathrm{Ti}, \mathrm{Li}\right)$

$\mathrm{T}=\left(\mathrm{Si}, \mathrm{Al}^{4+}, \mathrm{Fe}^{3+}, \mathrm{Ti}\right)$

The formula values of the cations were calculated based on 8 oxygen (Table 3). Based on the cationic values of $\mathrm{Al}$ and $\mathrm{Fe} /(\mathrm{Fe}+\mathrm{Mg})$, micas are divided into four groups: anite, siderophylite, phlogopite and eastonite (Spear, 1984). Among the end members of these groups, Si-Al and Fe-Mg cation exchange is common and the ratio of $\mathrm{Fe} /(\mathrm{Fe}+\mathrm{Mg})=0.33$ separates biotite mica from phlogopite. Based on the classification (Spear, 1984), the micas of the intrusive bodies from the northern side of the Shahroud River are located in the biotite field, between the poles of eastonite and siderophylite, and close to the pole of the eastonite (Figure 6-a). According to the classification diagram of Foster (1960), these biotites are magnesium biotites and phlogopites. The core and middle of them are type of iron-rich biotites and their margins are type of magnesium-rich (phlogopite) (Figures 6-b and c).
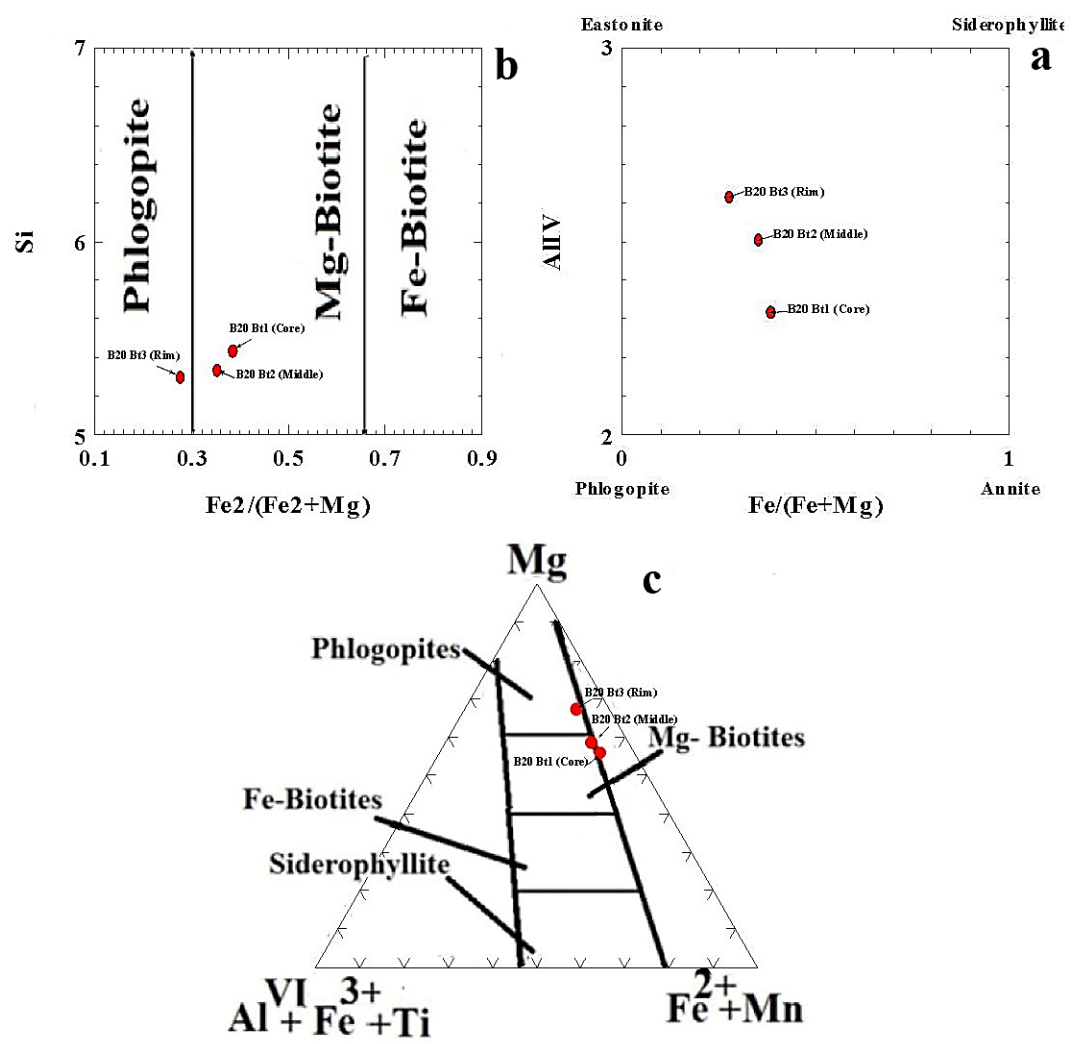

Figure 6. Chemical compositions of biotites in the study area on a) $\mathrm{Fe} /(\mathrm{Fe}+\mathrm{Mg})$ diagram versus $\mathrm{Al}^{+4}$ (Deer, 1991) b) $\mathrm{Fe} 2 /(\mathrm{Fe} 2+\mathrm{Mg}$ ) diagram versus $\mathrm{Si}$ c) $\mathrm{Mg}-\mathrm{Al}+6+\mathrm{Fe} 3++\mathrm{Ti}-\mathrm{Fe} 2++\mathrm{Mn}$ diagram (Foster, 1960). 


\section{Plagioclase}

The chemical formula of plagioclase was calculated based on 5 cations and 8 oxygen (Table 4). The results of microprobe analysis show that the composition of plagioclases of gabbro and olivine gabbro in the studied samples in different samples are such that it change from andesine to labrodorite, while their compositions in porphyry monzonite and hornfels calc silicate are oligoclase and albite, respectively (Figure $7 \mathrm{a}$ to $\mathrm{d}$ ). In most of the intrusive rocks on the northern side of Shahroud River, plagioclase minerals has zoning, so that in olivine gabbros, plagioclase has oscillating or reverse zoning (Figures 8- a and b), but in gabbro and porphyry monzonite, zoning is normal (Figures 8-c, d, e). It seems that changes in the amount of magma water (due to escape from the magma chamber) as well as contamination with limestone in the early stages of differentiation have caused oscillating and reverse zoning in olivine gabbro, while with the progressing of differentiation, lack of contamination and escape of water from the magmatic chamber have caused normal zoning in plagioclase of gabbro and monzonite porphyry.

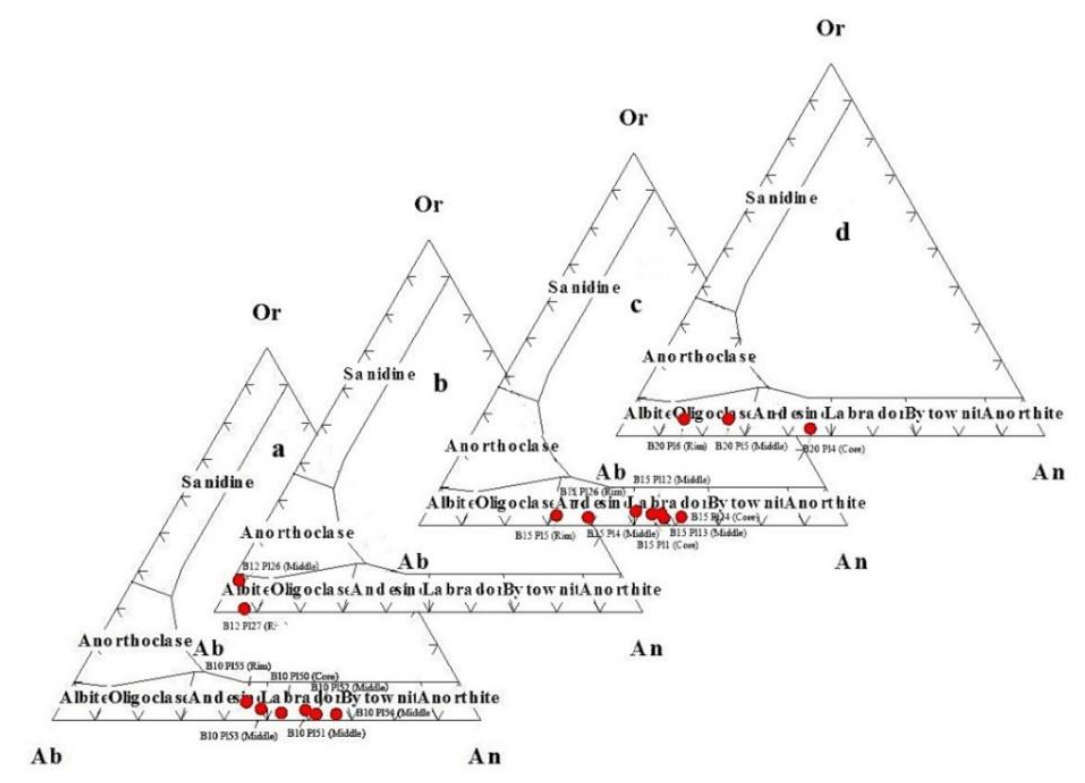

Figure 7. Chemical composition of plagioclase on the Or-Ab-An ternary diagram (Deer, 1991) a: olivine gabbro, b: calcsilicate hornfels, c: gabbro, d: monzonite porphyry of the study area.

\section{Alkali feldspar}

Alkali feldspars are essentially a series of solid solutions between $\mathrm{NaAlSi}_{3} \mathrm{O}_{8}$ and $\mathrm{KAlSi}_{3} \mathrm{O}_{8}$ that naturally contain very small amounts of $\mathrm{CaAl}_{2} \mathrm{Si}_{2} \mathrm{O}_{8}$ in solid solution. Anorthite content is generally less than $5 \%$ for the compositions between $\mathrm{Ab}_{100} \mathrm{Or}_{0}$ and $\mathrm{Ab}_{60} \mathrm{Or}_{40}$, but slightly higher in richer members than in sodium series (Figure 9a, b). Other ions that may be present in limited amounts include $\mathrm{Fe}^{3+}, \mathrm{Mg}, \mathrm{Sr}, \mathrm{Ba}, \mathrm{Ti}, \mathrm{Fe}^{2+}$, and to a much lesser extent $\mathrm{Mn}$. The general formula of alkali feldspar is as follows.

$(\mathrm{K}, \mathrm{Na}, \mathrm{Ca})\left[\mathrm{AlSiO}_{8}\right]$

The results of microprobe data show that the type of alkaline feldspars of intrusive rocks is orthoclase. The formula values of orthoclase ions were calculated based on 8 oxygen (Table 5). 


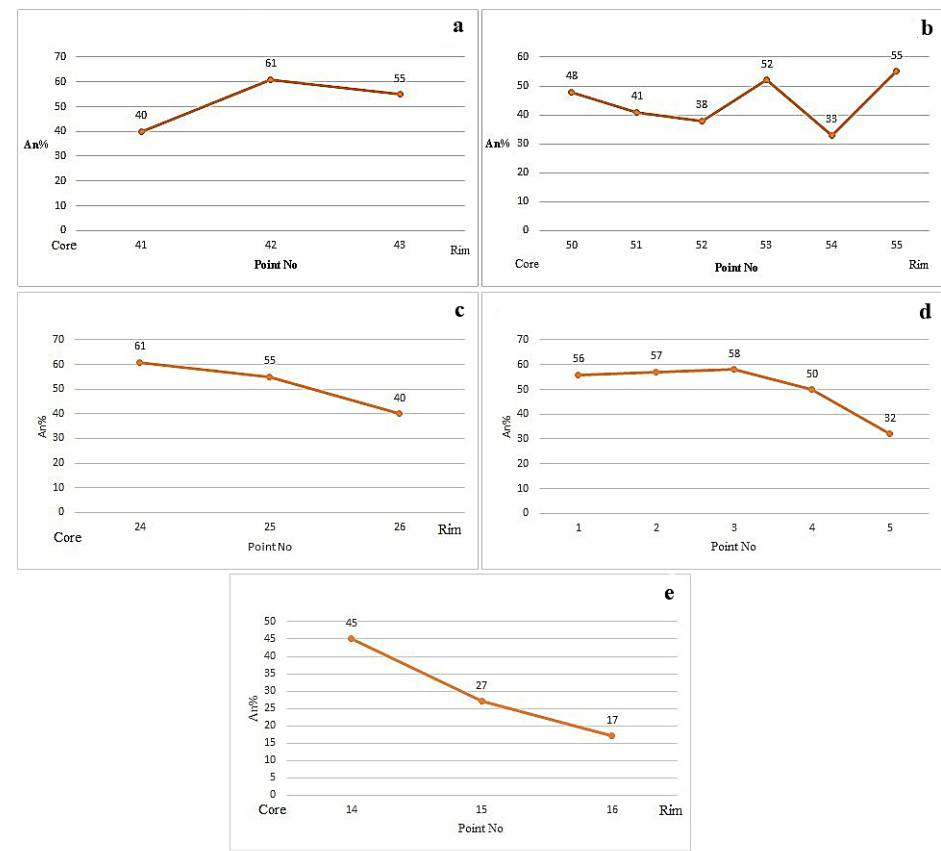

Figure 8. Histogram showing the compositional changes from center to margin in two samples of plagioclases in a \& b) olivine gabbro, c \& d) gabbro and e) porphyry monzonite. As can be seen, the zoning of plagioclase is oscillating or reverse in olivine gabbro, while in gabbro and monzonite porphyry is normal.

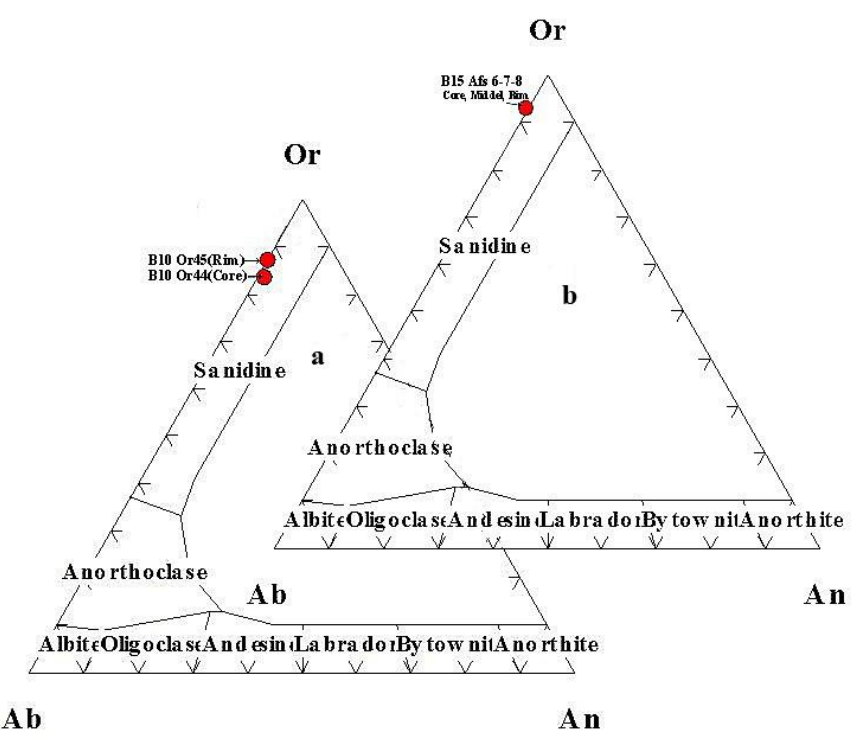

Figures 9-a and $\mathrm{b}$. The chemical composition of alkali feldspars present in the olivine-gabbro and gabbro in the study area, respectively in the triangle diagram of Or-Ab-An (Deer, 1991).

\section{Oxides}

The compositions of iron and titanium oxides in the region in terms of percentage in moles were transferred on the triangular diagram $\mathrm{FeO}-\mathrm{TiO}_{2}-\mathrm{Fe}_{2} \mathrm{O}_{3}$ (Deer et al, 1991) (Figure 10). As can be seen (Figure 10), the compositions of the core and the middle of the oxides of iron and titanium oxides are 
ilmenite type and but the margin is wostite. At the same time, the composition of iron and titanium oxides is such that it corresponds to a series of solid solutions formed over than $800{ }^{\circ} \mathrm{C}$ (Table 6 and Figure 10).

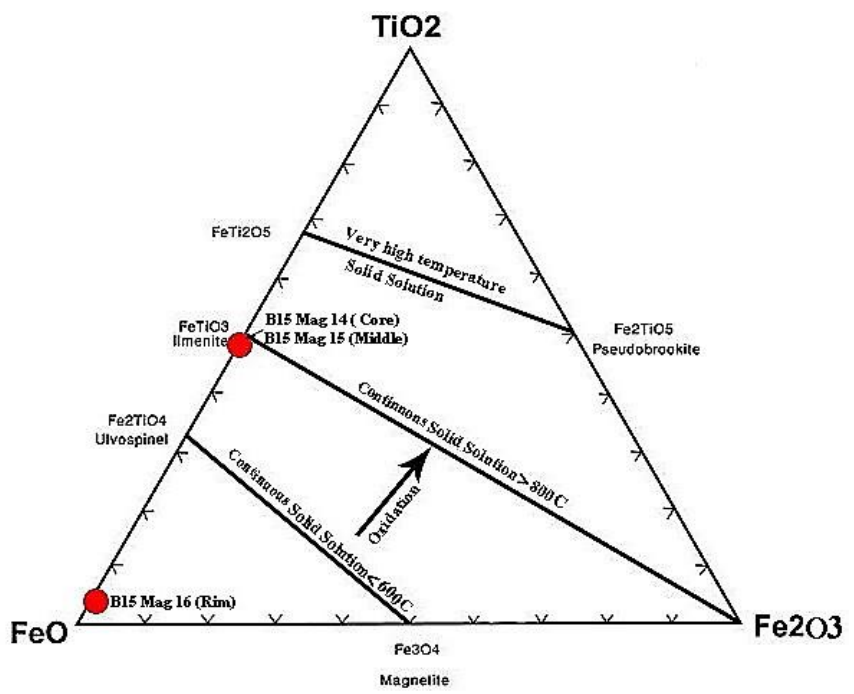

Figure 10. Chemical compositions of iron and titanium oxide on a triangular diagram of FeO-TiO2$\mathrm{Fe} 2 \mathrm{O} 3$ molecular ratios (Deer et al, 1991).

\section{Olivine}

In order to distinguish and study of olivines, figure 11 has been used. As can be seen in the $\mathrm{Fa}-\mathrm{Fo}$ diagram (Deer et al., 1992) (Figure 11), the olivine compositions of the intrusive bodies on the northern side of the Shahroud River are located in the hyalosidrite field. The microprobe data show that the olivines are composed in such a way that their margins are iron rich while their cores are more magnesium rich (normal zoning).

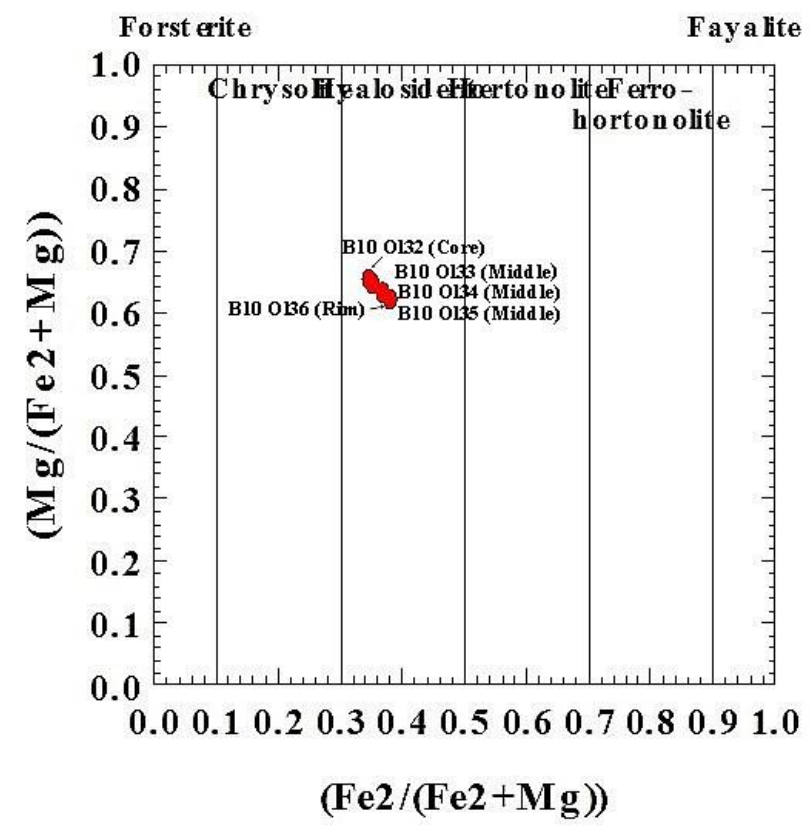

Figure 11. Olivine compositions in intrusive rocks from the north side of Shahroud River based on Fe2 + / $(\mathrm{Fe} 2++\mathrm{Mg})$ versus $\mathrm{Mg} /(\mathrm{Fe} 2++\mathrm{Mg})$ diagrams (Deer et al., 1992). 


\section{Pressure and temperature evaluation}

In this section, an attempt has been made to evaluate the temperature and pressure of rock formation in the study area using the chemical composition of the minerals forming the intrusive rocks in the study area and using conventional geothermometric and geobarometric methods.

\section{Amphibole barometer}

To find the pressure of rock formation in the area, we have used the amphibole geobarometer. Schmidt (1992) presented the following equation to calculate the pressure of amphibole formation.

$\mathrm{P}( \pm 0.6 \mathrm{Kbar})=-3.01+4.76 \mathrm{Al}^{\mathrm{tot}} \mathrm{Amp}$

In this equation, $\mathrm{Al}^{\text {tot }} \mathrm{Amp}$ is the total amount of aluminum in amphibole formula. The results of using of this barometer have been shown in table 6 for the samples from the study area.

Table 6. Evaluated pressures for amphiboles of samples form study area using Schmidt (1992) method.

\begin{tabular}{|c|c|}
\hline Lithology & (Pressure) P (Kbar) \\
\hline \multirow{3}{*}{ Monzonite porphyry } & 6.23 \\
\cline { 2 - 2 } & 5.51 \\
\cline { 2 - 2 } & 6.16 \\
\hline
\end{tabular}

\section{Thermometry and barometry based on pyroxene composition}

The chemical composition of pyroxenes is an important tool for measuring the temperature of igneous rocks. For the formation temperature of pyroxenes, XPT and YPT indices (Figure 12-a) are used, which are based on the following equations (Soesoo, 1997):

1) $\mathrm{XPT}=0 / 446 \mathrm{SiO} 2+0 / 187 \mathrm{TiO} 2-0 / 404 \mathrm{Al} 2 \mathrm{O} 3+0 / 346 \mathrm{FeO}-0 / 052 \mathrm{MnO}+0 / 309 \mathrm{Mgo}+0 / 446$ $\mathrm{CaO}-0 / 446 \mathrm{Na} 2 \mathrm{O}$

2) $\mathrm{YPT}=-0 / 369 \mathrm{SiO} 2+0 / 535 \mathrm{TiO} 2-0 / 317 \mathrm{Al} 2 \mathrm{O} 3+0 / 232 \mathrm{FeO}+0 / 235 \mathrm{MnO}-0.516 \mathrm{MgO}-$ $0 / 167 \mathrm{CaO}-0 / 153 \mathrm{Na} 2 \mathrm{O}$

An important advantage of this method in thermometry is that, firstly, the simultaneous presence of two pyroxenes is not required and secondly, it can be used for $\mathrm{Mg}-\mathrm{Ca}-\mathrm{Fe}$ and $\mathrm{Fe}-\mathrm{Mg}$ pyroxenes. According to this method, the formation temperature of pyroxenes in the intrusive bodies from the northern side of Shahroud River is 1100 to $1200{ }^{\circ} \mathrm{C}$. Also, using the pyroxene crystallization temperature diagram (Lindsly, 1983) for a pressure of $5 \mathrm{kbar}$, a temperature range of 650 to $1100^{\circ} \mathrm{C}$ is obtained for clinopyroxenes of the study area. The orthopyroxenes in the intrusives from the north side of the Shahroud River have crystallized in the temperature range between 1000 and $1100{ }^{\circ} \mathrm{C}$, and it seems that clinopyroxenes have continued to crystallize at lower temperatures than orthopyroxenes (Figure 12-c). In order to determine the pressure using the diagram of (Soesoo, 1997) and using the values of XPT and YPT according to (Figure 12-b), the crystallization pressure of clinopyroxenes is estimated from $>2$ to about 8 kbar. 

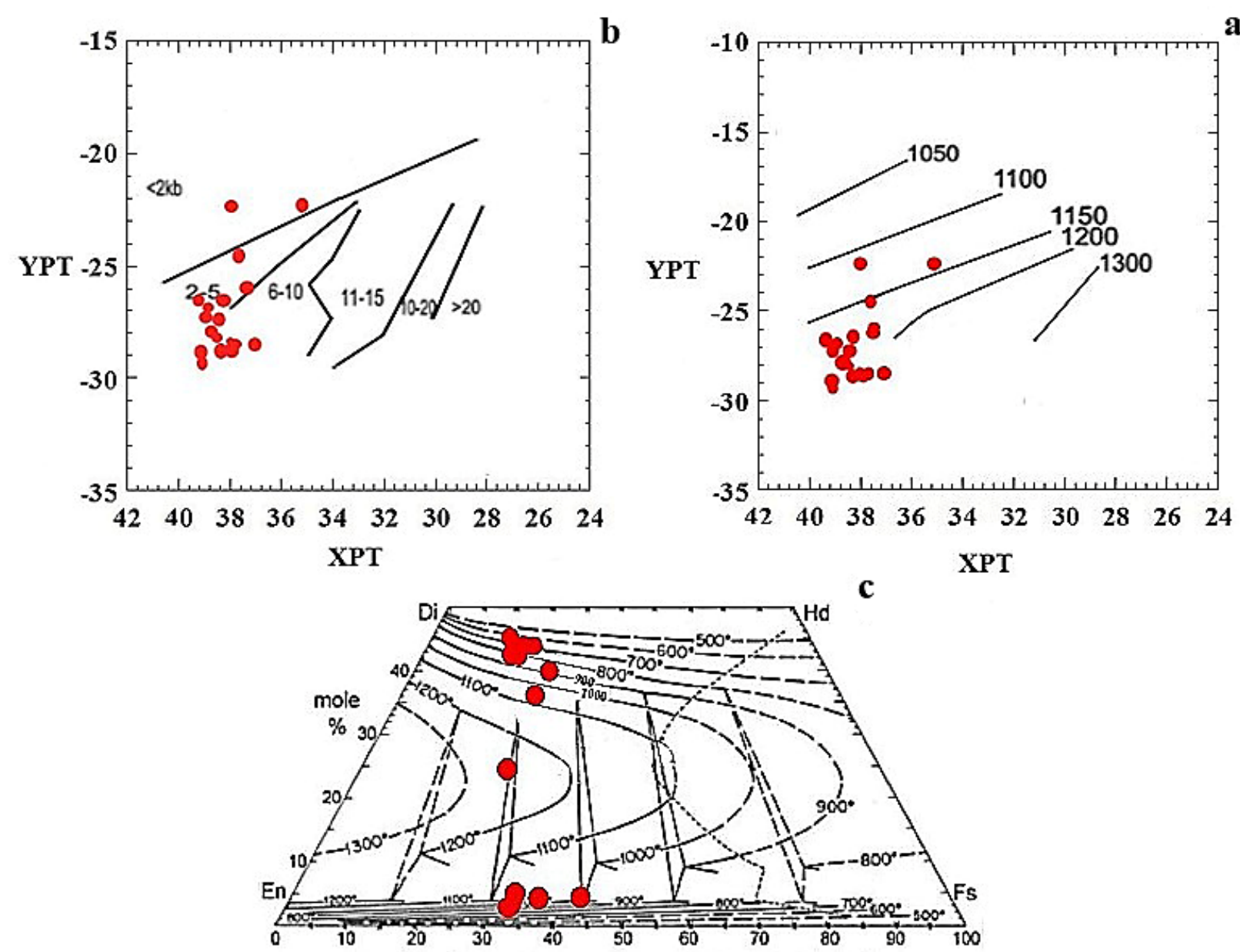

Figures 12. a and b: Diagrams of temperature and pressure formation of clinopyroxenes in the intrusions of the northern side of Shahroud river using (Soesoo, 1997) method, the formation temperature of clinopyroxenes is between 1100 and $1200{ }^{\circ} \mathrm{C}$ at pressures of 2 to 5 kbar. c) Determining the formation temperature of clinopyroxenes in the intrusions of the northern side of Shahroud River using a single pyroxene thermometer (Lindsly, 1983).

\section{Feldspar thermometer}

The thermometer proposed by Stormer (1975) has been used to measure the formation temperature of those feldspar rocks in the area that contain both plagioclase and feldspar alkali. The Ab-An-Or triangular diagram was used to determine the crystallization temperature and pressure of feldspars (Figure 13). According to this diagram, the temperature of the feldspars was 750 and the required pressure was 1 kilobar. 


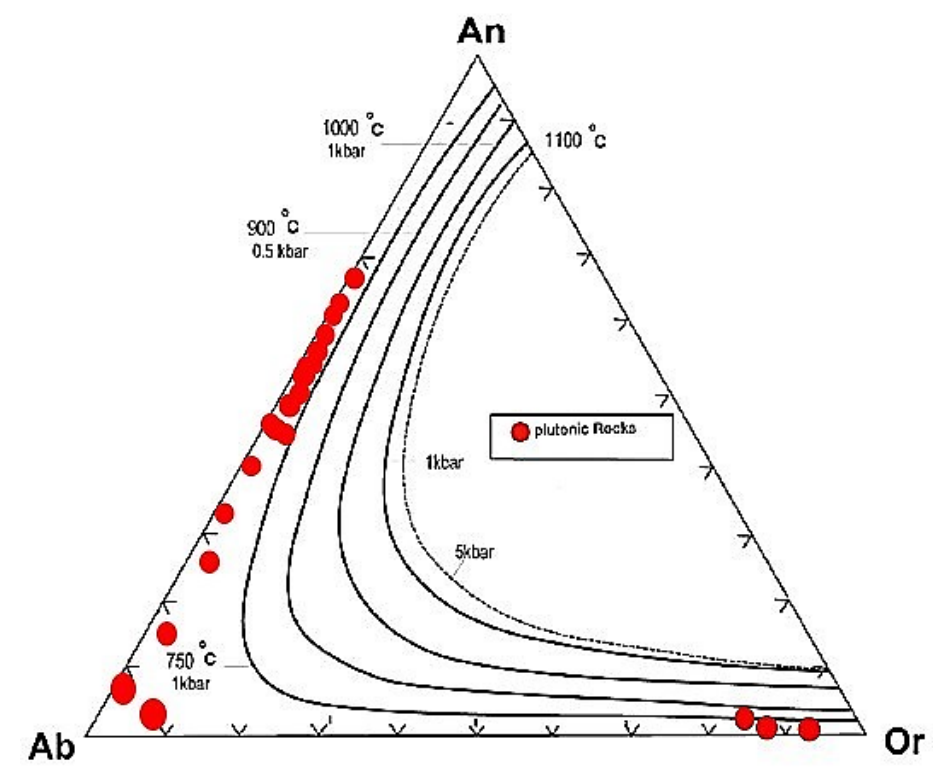

Figure 13. Or-An-Ab triple diagram (Koroll et al., 1993). Isotherms at $750{ }^{\circ} \mathrm{C}$ and a pressure of $1 \mathrm{kbar}$, $900^{\circ} \mathrm{C}$ and a pressure of $0.5 \mathrm{kbar}, 1000^{\circ} \mathrm{C}$ and a pressure of $1 \mathrm{kbar}, 1100^{\circ} \mathrm{C}$ and a pressure of 1 to 5 kbar for the crystallization of feldspars. The plagioclase in the samples of the study area, according to this diagram, shows a temperature of $750^{\circ} \mathrm{C}$ and a pressure of $1 \mathrm{kbar}$.

\section{Magmatic series and tectonic setting}

The wide range of chemical composition of clinopyroxenes in various igneous rocks can indicate the origin, nature and tectonic setting of the magma that forms these minerals. So the chemical composition of clinopyroxenes is a function of the environment conditions and the chemical composition of their constituent magma (Le Bas, 1962). Using microprobe data of these minerals and using the $\mathrm{SiO} 2-\mathrm{Al} 2 \mathrm{O} 3$ diagram (Nisbet et al, 1977) (which is able to distinguish between high-alkaline, alkaline and sub-alkaline magmas), we obtained the magmatic series of intrusive rocks in the study area. This diagram shows that the magmatic series is of the sub-alkaline type (Figure 14-a).

One of the diagrams that can seperate the types of tectonomagmatic environments is F1 - F2 diagram (Leterrier et al., 1982). The values F1 - F2 are obtained using formulas 1 and 2.

Formula (1)

$\mathrm{F} 1=-0.012 * \mathrm{SiO}_{2}-0.0807 * \mathrm{TiO}_{2}+0.0026 * \mathrm{Al}_{2} \mathrm{O}_{3}-0.0012 * \mathrm{FeO} *-0.0026 * \mathrm{MnO}+0.0087 * \mathrm{MgO}-0.0128 * \mathrm{CaO}-$ $0.0419 * \mathrm{Na}_{2} \mathrm{O}$

Formula (2)

$\mathrm{F} 2=-0.0469 * \mathrm{SiO} 2-0.0818 * \mathrm{TiO} 2-0.0212 * \mathrm{Al} 2 \mathrm{O} 3-0.0041 * \mathrm{FeO} *-0.1435 * \mathrm{MnO}-$ $0.0029 * \mathrm{MgO}+0.085 * \mathrm{CaO}+0.0160 * \mathrm{Na} 2 \mathrm{O}$

The results show that the tectonomagmatic environment of the magma is volcanic arc type (Figure 14-c, b). 

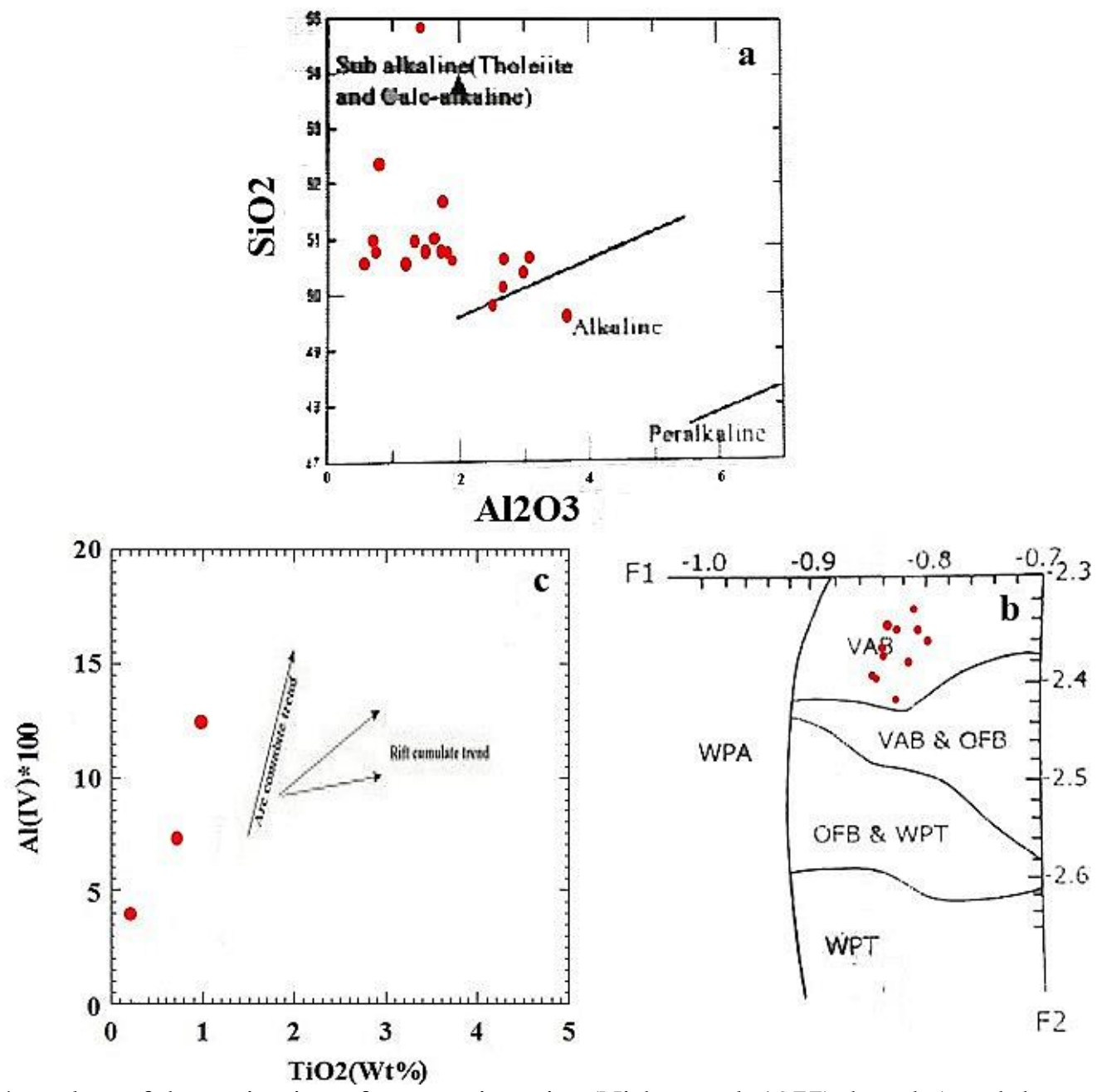

F2

Figure 14-a. plots of determination of magmatic series (Nisbet et al, 1977), b and c) and determination of tectonomagmatic environment (Le Bas, 1962) and Leterrier et al., 1982 using the results of microprobe analysis of clinopyroxenes.

\section{CONCLUSION}

Using microprobe analysis data and based on mineral chemistry and related diagrams, the following results were obtained:

- The chemical composition of amphiboles in the study area in the general diagram of amphiboles is in the field of calcium amphiboles and their composition is of the type of pargasite and ferropargasite. At the same time, these amphiboles have zoning so that their core composition is ferropargasite (ferrohornblende) and the middle and marginal parts are pargasite (hornblende).

- Pyroxenes, according to the Q-J diagram, are all in the realm of calcium-magnesium-iron pyroxenes and their type is augite, diopside and hypersthene. The resulting data show that the composition of augites is such that their margins are magnesium rich while their core are ironcalcium rich, but hypersthens, they have magnesium rich cores and iron rich margins.

- Biotites have magnesium biotite and phlogopite compositions.

- The composition of plagioclase in gabbro and olivine gabbro varies from andesine to labradorite, while its composition in monzonite porphyry and calcsilicate hornfels is oligoclase and albite, 
respectively. At the same time, in olivine gabbro, plagioclase has oscillating or reverse zoning, but in gabbro and monzonite porphyry, zoning is normal. It seems that changes in the amount of magma water (due to escape from the magma chamber) as well as contamination with limestone in the early stages of differentiation have caused oscillating and reverse zoning in olivine gabbro. While with the progressing of differentiation, lack of contamination and lack of escape of water from the magmatic chamber has caused plagioclase in gabbro and porphyry monzonite to have normal zoning.

- Alkaline feldspars are orthoclases.

- Olivines have a hyalosideritic composition with zoning so that their margins have more iron while their core have more magnesium (normal zoning).

- The core and middle composition of iron and titanium oxide crystals are ilmenite and but their margins are wostite.

- According to the results obtained from the amphibole geobarometer, monzonite rocks are formed at a pressure between 5.51 and 6.23. Pyroxene geobarometer shows that the crystallization of clinopyroxenes in the intrusive rocks of the region has taken place at pressures between $>2$ to about $5 \mathrm{kbar}$. Also, using the pyroxene crystallization temperature diagram, a temperature range of 650 to $1100^{\circ} \mathrm{C}$ is obtained for clinopyroxenes in the intrusive bodies from the northern side of the Shahroud River. The orthopyroxenes in the intrusions from the northern side of the Shahroud River have crystallized in the temperature range between 1000 and $1100{ }^{\circ} \mathrm{C}$, so it seems that clinopyroxenes have continued to crystallize at lower temperatures than orthopyroxenes.

- Based on the magma series and tectonomagmatic descremination diagrams using a composition of clinopyroxenes, the magma is of the subalkaline type and was formed in a volcanic arc environment.

\section{REFERENCES}

Baharfiruzi, K., Shafei, A. (2003). Geological 1:100000 scale map and report of Javaherdeh. Geological Survey of Iran, Iran.

Deer, W. A., Howie, R. A., Sussman, J. (1992). An introduction to the rock forming minerals. Longman Ltd 528p.

Foster, M. D. (1960). Interpretation of the composition of trioctahedral micas. United States Geological Survey Professional Paper, 354-B, 11-46.

Ghalamghash, J., Rashid, H. (2002). Geological 1:100000 scale map and report of Jirandeh. Geological Survey of Iran, Iran.

Hawthorne, F. C. (1981). Crystal Chemistry of the Amphiboles. Canadian Mineralogist, 21, 173-480.

Hou, T., Zhang, Z. C., Keiding, J. K., Veksler, I. V. (2015). Petrogenesis of the ultrapotassic Fanshan intrusion in the North China Craton: implications for lithospheric mantle metasomatism and the origin of apatite ores. Journal of Petrology, 5, 893-918.

Jacamon, F., Larsen, R. B. (2009). Trace element evolution of quartz in the charnockitic Kleivan granite, SWNorway: The Ge/Ti ratio of quartz as an index of igneous differentiation. Lithos 107, 281-291.

Leake, B. E., Woolly, A. R., Arps, C. E. S., Birch, W.D., Gilbert, M. C., Grice, J.D., Hawthorne, F. C., Kato, A., Kisch, H. J., Krivovichev, V. G., Linthout, K., Laird, J., Mandarino, J., Maresch, W. V., Nickel, E. H., Rock, N. M. S., Schmucher, J. C., Smith, D. C., Stephenson, N. C. N, Unungaretti L., Whittaker, E.J.W., Youzhi G., (1997). 
Nomenclature of amphiboles. Report of the Subcommittee on Amphiboles of the International Mineralogical Association Commission on New Minerals Names, Europian Journal of Mineralogy, 9, 623-651.

Le Bas M. J. (1962). The role of aluminium in igneous clinopyroxenes with relation to their percentage, American Journal of Sciences, 88, 260-267.

Leterrier, J., Maury, R. C., Thonon, P., Girard, D., Marchal, M. (1982). Clinopyroxene composition as a method of identification of the magmatic affinities of paleo-volcanic series.Earth and Planetary Science Letters, 59 (1), 139154.

Liang, Y., Deng, J., Liu, X., Wang, Q., Qin, C., Li, Y., Yang, Y., Zhou, M., Jiang, J. (2018). Major and trace element, and $\mathrm{Sr}$ isotope compositions of clinopyroxene phenocrysts in mafic dykes on Jiaodong Peninsula, southeastern North China Craton: Insights into magma mixing and source metasomatism. Lithos, 302-303, 480-495.

Lindsly, D. H. (1983). Pyroxene geothermometry. American Mineralogist, 68, 477-493.

Morimoto, N., Fabrices, J., Ferguson, A. K., Ginzburg, I. V., Ross, M., Seifer, F. A., Zussman J., Akoi, K., Gottard, G. (1988). Nomenclature of pyroxenes", Mineralogical Magazine. 52, 535-550.

Nisbet, E. G., Pearce, J.A. (1977). Clinopyroxene composition in mafic lavas from different tectonic settings, Contribution to mineralogy and petrology, 63, 149-160.

Nogole-Sadat, M. A., Almasian, M. (1993). Tectonic map of Iran. Geological Survey of Iran, Tehran.

Ovung, T. N., Ray, J., Ghosh, B., Koeberl, C., Topa, D., Paul, M. (2017). Clinopyroxene composition of volcanics from the Manipur Ophiolite Northeastern India: implications to geodynamic setting. International Journal of Earth Sciences, DOI 10.1007/s00531-017-1529-y.

Poldervaart, A., Hess, H. H. (1951). Pyroxenes in crystallisation of basaltic magmas. Journal of geology, 59, 472489.

Soesoo, A. A. (1997). Multivariate statistical analysis of clinopyroxene composition: empirical coordinates for the crystallisation PT-estimations. Geological Society of Sweden (Geologiska Föreningen), 119, 55-60. 